\title{
On the Convergence of the Quasi-Periodic Approximations on a Finite Interval
}

\author{
A. Poghosyan, L. Poghosyan, and R. Barkhudaryan
}

\begin{abstract}
We investigate the convergence of the quasi-periodic approximations in different frameworks and reveal exact asymptotic estimates of the corresponding errors. The estimates facilitate a fair comparison of the quasi-periodic approximations to other classical well-known approaches. We consider a special realization of the approximations by the inverse of the Vandermonde matrix, which makes it possible to prove the existence of the corresponding implementations, derive explicit formulas and explore convergence properties. We also show the application of polynomial corrections for the convergence acceleration of the quasi-periodic approximations. Numerical experiments reveal the auto-correction phenomenon related to the polynomial corrections so that utilization of approximate derivatives surprisingly results in better convergence compared to the expansions with the exact ones.
\end{abstract}

Key Words: Truncated Fourier series, convergence acceleration, quasi-periodic interpolation, quasi-periodic approximation

Mathematics Subject Classification 2010: 42A10, 65T40

\section{Introduction}

We continue investigations of the reconstruction problem of a smooth but non-periodic function $f$ on $[-1,1]$ by the finite number of its Fourier coefficients

$$
f_{n}=\frac{1}{2} \int_{-1}^{1} f(x) e^{-i \pi n x} d x,|n| \leq N .
$$

It is well known that the solution of the problem by the truncated Fourier series or trigonometric interpolation via corresponding Fourier coefficients is ineffective for non-periodic functions due to slow pointwise convergence 
on $(-1,1)$ and the Gibbs phenomenon at the endpoints (see $[1,5]$ ). The degraded convergence may imply an impression that the accurate reconstruction of $f$ from the knowledge of the coefficients is impossible. However, many authors proved the opposite by showing that the spectral information is sufficient even for non-periodic functions. There is ample literature devoted to the resolution of the Gibbs phenomenon and convergence accelerations. It is impossible to summarize such a great amount of work. Individual articles include 6 [56]. We will follow only the ideas related to the current paper.

An efficient approach to convergence acceleration by subtracting a polynomial representing the discontinuities (jumps) in the function and its derivatives was suggested by Krylov [57] (see also [58]). Lanczos [59] independently developed the same approach by introducing the basic system of 2-periodic Bernoulli polynomials. We will refer to this approach as the Krylov-Lanczos method. Jones and Hardy [60] and Lyness [61] considered convergence acceleration of trigonometric interpolations by the polynomial subtraction (see also 62, 63]).

The key problem in the Krylov-Lanczos method is approximation of the jumps. In the case of interpolations, as the pointwise values of the function are known, the finite difference formulas can be applied for approximation of the derivatives in jumps. Approaches resembling this approach have been attempted in a series of papers $[59,61,64,66]$. However, the approximation of jumps via finite differences is not recommended due to numerical instability (see for example [61]). Even in the case of a uniform grid, finite difference approximations are notoriously unreliable. Moreover, in many applications, the pointwise values are not explicitly available.

The first attempt towards a more robust approach was initiated in $67-$ 73]. The general approach was established by Eckhoff in a series of papers 74 76, showing that coefficients contain sufficient information to reconstruct the jump-values. We will refer to this approach as the Eckhoff method, where the fundamental aspect is the approximation of the jumps by the solution of a linear system of equations. A similar idea was used in the Richardson extrapolation process $[77]$. Investigations and generalizations of the Krylov-Lanczos and the Eckhoff methods see also in [78 89]. Polynomial corrections applied to trigonometric expansions for the approximation/interpolation of the piecewise-smooth functions completely eliminate the Gibbs phenomenon and accelerate convergence both away from the singularities and on the entire interval. The main difficulty of its realization is the detection of singularities and the approximation of the corresponding jumps.

Another approach that does not eliminate the Gibbs phenomenon but mitigates its impacts utilizes expansions with trigonometric functions whose periods are larger than the lengths of approximation or interpolation in- 
tervals. The idea of such expansions was introduced in [90]. It considered trigonometric interpolation of a function $f$ on $[-1,1]$ for the following basisfunctions

$$
\left\{e^{i \pi n \sigma x}\right\},|n| \leq N, \sigma=\frac{2 N}{2 N+m+1}, m=0,1,2, \ldots,
$$

and for the grid $x_{k} \in[-1,1]$,

$$
x_{k}=\frac{k}{N},|k| \leq N,
$$

that includes the endpoints of the interval. All basis functions have the period $2 / \sigma$, which tends to 2 (the length of the interval of interpolation) as $N$ tends to infinity.

That is why those expansions were called as quasi-periodic (QP-) interpolations. Let us introduce the idea of [90] in more details (see also [91]). Consider a new function $g(t)$ defined on $[-\sigma, \sigma]$ by the following change of variable

$$
g(t)=f\left(\frac{t}{\sigma}\right)=f(x), x \in[-1,1], t \in[-\sigma, \sigma], t=\sigma x .
$$

This implies interpolation of $g(t)$ on the grid

$$
t_{k}=\sigma x_{k}=\frac{2 k}{2 N+m+1},|k| \leq N
$$

Thus, the QP-interpolation actually interpolates $g(t)$ on the grid $t_{k}$ and is exact for $e^{i \pi n t},|n| \leq N$. It is important to note that for $m>0$, the 2-periodic extension of the grid $t_{k}$ to the real line is non-uniform. This non-uniformity should be one of the main reasons of better convergence properties of the QP-interpolation compared to the classical trigonometric interpolation that uses uniform grids.

Papers [91 95] investigated the pointwise convergence of the QP-interpolation and its convergence in the $L_{2}$-norm. The application of polynomial corrections to the QP-interpolation was considered in [95 for complete elimination of the Gibbs phenomenon and additional convergence acceleration. Similar ideas were applied to the truncated Fourier series in [96]. The corresponding expansions were named as QP-approximations. The main idea was the extension of a function $f$ from the interval $[-1,1]$ onto the larger interval $[-1 / \sigma, 1 / \sigma]$ and its approximation via truncated Fourier series with quasi-periodic basis-functions. The main problem in the realization of the idea is the determination of the coefficients on the extended interval, where the function $f$ was unknown. Paper [96] described three algorithms $\mathrm{A}, \mathrm{B}$, and $\mathrm{C}$ that differently solved the problem. Moreover, it showed how the 
required coefficients could be approximated via classical Fourier coefficients known for the interval $[-1,1]$.

The main goal of the current paper is the investigation of the convergence of Algorithm C. The main benefit of the algorithm is its explicit realization that allows us to perform theoretical investigations regarding the existence of the solution and convergence properties. Further, we show how the polynomial correction method can be applied for additional convergence acceleration.

Approximations with non-periodic basis functions were also considered in 97 99]. However, they were essentially different by using the classical Fourier coefficients on $[-1,1]$ rather than the Fourier quasi-periodic coefficients on the extended interval.

Another approach that utilized non-periodic basis functions was introduced in [100,101]. Here, the truncated Fourier series by the following modified trigonometric system was considered

$$
\mathcal{H}=\left\{\cos \pi n x: n \in \mathbb{Z}_{+}\right\} \cup\left\{\sin \pi\left(n-\frac{1}{2}\right) x: n \in \mathbb{N}\right\}, x \in[-1,1] .
$$

It was originally proposed by [102 without investigation of its properties. System $\mathcal{H}$ is an orthonormal basis of $L_{2}[-1,1]$ as it consists of the eigenfunctions of the Sturm-Liouville operator

$$
\mathcal{L}=-d^{2} / d x^{2}
$$

with Neumann boundary conditions $f^{\prime}(1)=f^{\prime}(-1)=0$. The corresponding univariate and multivariate approximations were thoroughly investigated in a series of papers $[103-106]$. Some of the basis functions $\sin \pi\left(n-\frac{1}{2}\right) x$ are nonperiodic on $[-1,1]$. The rest of the basis functions $\cos \pi n x$ are 2-periodic. The non-periodicity implies some slight improvements in convergence compared to the classical Fourier series on $[-1,1]$. However, the convergence rate of the modified Fourier approximation remains relatively slow. If $N$ is the truncation parameter, the uniform error is $O\left(N^{-1}\right)$ on $[-1,1]$ and $O\left(N^{-2}\right)$ away from the endpoints. Better convergence results were achieved only via application of polynomial corrections [107,108 or rational trigonometric functions 109 111. Interpolations by the modified trigonometric system were considered in [112]. Similarly, the convergence rate compared to the classical trigonometric interpolation was improved. Overall, without additional convergence acceleration, the expansions via modified trigonometric system have worse convergence compared to the QP-approximations and interpolations. One of our future works will be application of the ideas of the quasi-periodic expansions to the modified trigonometric system.

The idea of non-periodic approximations or interpolations is not new. Many authors considered similar ideas of approximation on extended domains $[-T, T], T>1$. Thus, they sought an approximation $F_{N}(f)$ to $f$ 
from the set

$$
\mathcal{G}_{N}:=\operatorname{span}\left\{\varphi_{n}:|n| \leq N\right\}, \varphi_{n}(x)=\frac{1}{\sqrt{2 T}} e^{i \frac{\pi n}{T} x} .
$$

Papers 113 115 proposed to compute $F_{N}(f)$ as the best approximation to $f$ on $[-1,1]$ in a least squares sense (named as continuous extension):

$$
F_{N}(f):=\operatorname{argmin}_{\phi \in \mathcal{G}_{N}}\|f-\phi\|,
$$

where $\|\cdot\|$ is the standard norm on $L_{2}(-1,1)$. In [115 117] it was shown that this approach leads to geometrically fast approximations for analytic functions. Regarding parameter $T$, those papers recommended $T=2$ as the best choice, and also

$$
T=\frac{\pi}{4}\left(\arctan \left(\left(e_{t o l}\right)^{\frac{1}{2 N}}\right)\right)^{-1}
$$

for oscillatory functions, where $e_{t o l}<<1$. The latest resembles some similarity to our approach as parameter $1 / \sigma=T$ also tends to 1 as $N \rightarrow \infty$. However, the constructions are essentially different from the QP-approximations by solving different linear systems of equations and utilizing different frameworks for optimality. One of our future works will be devoted to the convergence analysis of analytical functions and comparison with those known results. Another important topic should be stability analysis of the QPapproximations and comparison with the stabilities of known methods.

More general problem was considered in [118,119] dealing with the reconstruction of piecewise-smooth functions on a finite interval via Fourier data. This is the most general approach, where the points of discontinuities with the corresponding jumps are unknown. Similar problem with combination of the QP-approximations will be considered elsewhere. In the current paper, we explore the simplest case with a discontinuity point at the endpoints of the interval (the case of non-periodic function), where the only problem is the determination of the corresponding jumps according to the ideas of Eckhoff.

The paper is organized as follows. Section 1 introduces QP-approximations and specifically Algorithm C. Section 2 proves some preliminary results. Section 3 explores the pointwise convergence away from the endpoints and at $x= \pm 1$. Section 4 deals with $L_{2}$-convergence on the entire interval. In both cases, we derive exact asymptotic estimates for the corresponding errors. Section 5 considers convergence acceleration of the QP-approximations via polynomial corrections. It also studies the methods of approximation of function derivatives at the endpoints of the interval. It shows that application of approximate derivatives improves the convergence properties compared to the expansions that use the exact values of the derivatives. This improvement is known as auto-correction phenomenon which was previously detected and explained for polynomial corrections applied to trigonometric approximations and interpolations 86,87 . 


\section{Quasiperiodic Approximations}

Let a function $f$ be defined on $[-1,1]$. The main idea of the QP-approximations 96 is the extension of $f$ from $[-1,1]$ onto the larger interval $[-1 / \sigma, 1 / \sigma]$ and the application of the truncated Fourier series with the quasi-periodic basis functions

$$
S_{N, m}(f, x)=\sum_{n=-N}^{N} F_{n, m} e^{i \pi n \sigma x}, x \in[-1,1],
$$

where

$$
F_{n, m}=\frac{\sigma}{2} \int_{-\frac{1}{\sigma}}^{\frac{1}{\sigma}} f^{*}(x) e^{-i \pi n \sigma x} d x
$$

and $f^{*}$ stands for the extension of $f$. Note that $m=-1$ corresponds to the truncated Fourier series of $f$ on the interval $[-1,1]$. It is essential to observe that $\sigma$ in Equation (6) depends on $N$ and $m$. It means that the coefficients $F_{n, m}$ not only depend on $m$ but also on $N$. For different values of $N$, the entire set of coefficients $\left\{F_{n, m}\right\}_{n=-N}^{N}$ should be recomputed.

Calculation of the coefficients $F_{n, m}$ requires the knowledge of $f^{*}$ outside of $[-1,1]$. We put

$$
f^{*}(x)= \begin{cases}f_{\text {left }}(x), & x \in\left[-\frac{1}{\sigma},-1\right), \\ f(x), & x \in[-1,1] \\ f_{\text {right }}(x), & x \in\left(1, \frac{1}{\sigma}\right]\end{cases}
$$

and split the coefficients (6) into three terms

$$
F_{n, m}=\frac{\sigma}{2} \int_{-\frac{1}{\sigma}}^{-1} f_{l e f t}(t) e^{-i \pi n \sigma t} d t+f_{n, m}+\frac{\sigma}{2} \int_{1}^{\frac{1}{\sigma}} f_{\text {right }}(t) e^{-i \pi n \sigma t} d t,
$$

where

$$
f_{n, m}=\frac{\sigma}{2} \int_{-1}^{1} f(t) e^{-i \pi n \sigma t} d t
$$

We see that the main problem of the realization of the QP-approximations is the calculation of the first and the third integrals in the right-hand side of (7) as functions $f_{\text {left }}$ and $f_{\text {right }}$ are unknown.

Paper [96] considered three different approaches (Algorithms A, B and C) for the extensions. Algorithm A defines $f_{\text {left }}$ and $f_{\text {right }}$ explicitly as a linear combination of some "supporting" functions with unknown coefficients and considers a system of linear equations for their determination. Algorithms B and $\mathrm{C}$ perform implicit extension and approximate the mentioned integrals in (7) via some quadrature formulae. The unknown values of the left and 
right extensions on the corresponding grids of the quadratures can be found as a solution to a system of linear equations. Algorithm B applies Gaussian quadrature and Algorithm $\mathrm{C}$ uses the simplest rectangular quadrature.

Let us explain Algorithm $\mathrm{C}$ in more details. Let

$$
x_{j}^{*}=1+\frac{j}{2 N}+\frac{1}{4 N}, j=0, \ldots, m
$$

be the grid of the rectangular quadrature on $[1,1 / \sigma]$, and

$$
w_{j}=\frac{1}{2 N}, j=0, \ldots, m
$$

be the corresponding weights. The application of the rectangular rule to the first and the third integrals in the right-hand side of (7) implies

$$
F_{n, m}=\frac{\sigma}{2} \frac{1}{2 N} \sum_{j=0}^{m} f_{l e f t}\left(-x_{j}^{*}\right) e^{i \pi n \sigma x_{j}^{*}}+f_{n, m}+\frac{\sigma}{2} \frac{1}{2 N} \sum_{j=0}^{m} f_{\text {right }}\left(x_{j}^{*}\right) e^{-i \pi n \sigma x_{j}^{*}} .
$$

Denoting

$$
c_{j}^{\text {left }}(f)=\frac{\sigma}{2} \frac{1}{2 N} f_{\text {left }}\left(-x_{j}^{*}\right), c_{j}^{\text {right }}(f)=\frac{\sigma}{2} \frac{1}{2 N} f_{\text {right }}\left(x_{j}^{*}\right),
$$

we rewrite (8) as follows

$$
F_{n, m}=\sum_{j=0}^{m} c_{j}^{l e f t}(f) e^{i \pi n \sigma x_{j}^{*}}+f_{n, m}+\sum_{j=0}^{m} c_{j}^{r i g h t}(f) e^{-i \pi n \sigma x_{j}^{*}} .
$$

Paper 96 proposed determination of unknowns $\left\{c_{j}^{\text {left }}\right\}_{j=0}^{m}$ and $\left\{c_{j}^{\text {right }}\right\}_{j=0}^{m}$ from the following system of linear equations

$$
F_{n, m}=0,|n|=N-m, \ldots, N .
$$

We hoped that 10. would assure a smooth and periodic continuation outside of $[-1,1]$ resulting in accelerated convergence of the corresponding truncated Fourier series on the extended interval.

Throughout the paper, we only consider Algorithm $\mathrm{C}$ as it allows us to solve explicitly the system (10) through the inverse of a Vandermonde matrix. Let us derive it (see also $[96]$ ). For $\ell=0, \ldots, m$, we have

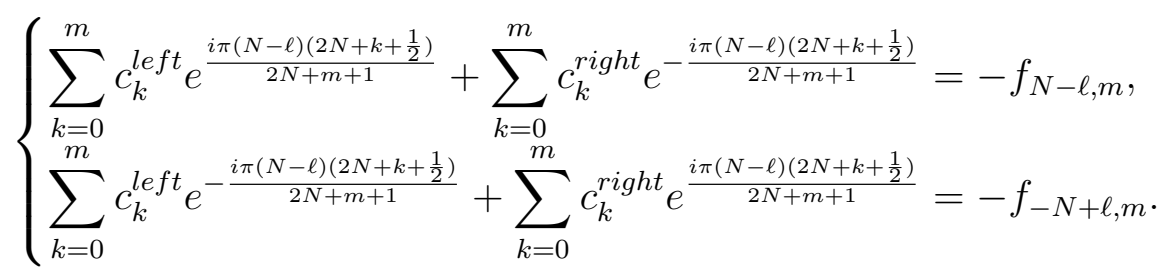


After changing the summation orders in the first sums, we get

$$
\left\{\begin{array}{l}
\sum_{k=m+1}^{2 m+1} c_{2 m+1-k}^{l e f t} e^{-\frac{i \pi(N-\ell)\left(2 N+k+\frac{1}{2}\right)}{2 N+m+1}}+\sum_{k=0}^{m} c_{k}^{r i g h t} e^{-\frac{i \pi(N-\ell)\left(2 N+k+\frac{1}{2}\right)}{2 N+m+1}}=-f_{N-\ell, m}, \\
\sum_{k=m+1}^{2 m+1} c_{2 m+1-k}^{l e f t} e^{\frac{i \pi(N-\ell)\left(2 N+k+\frac{1}{2}\right)}{2 N+m+1}}+\sum_{k=0}^{m} c_{k}^{r i g h t} e^{\frac{i \pi(N-\ell)\left(2 N+k+\frac{1}{2}\right)}{2 N+m+1}}=-f_{-N+\ell, m} .
\end{array}\right.
$$

Denoting

$$
c_{k}=c_{k}^{\text {right }}, k=0, \ldots, m, c_{k}=c_{2 m+1-k}^{\text {left }}, k=m+1, \ldots, 2 m+1,
$$

we rewrite the previous system as follows

$$
\left\{\begin{array}{l}
\sum_{\substack{k=0 \\
2 m+1}}^{2 m} c_{k} e^{-\frac{i \pi(N-\ell) k}{2 N+m+1}}=-e^{\frac{i \pi(N-\ell)\left(2 N+\frac{1}{2}\right)}{2 N+m+1}} f_{N-\ell, m}, \quad \ell=0, \ldots, m \\
\sum_{k=0}^{2 m+1} c_{k} e^{\frac{i \pi(N-\ell) k}{2 N+m+1}}=-e^{-\frac{i \pi(N-\ell)\left(2 N+\frac{1}{2}\right)}{2 N+m+1}} f_{-N+\ell, m}, \quad \ell=0, \ldots, m
\end{array}\right.
$$

with unknowns $\left\{c_{k}\right\}_{k=0}^{2 m+1}$ and with the following Vandermonde matrix

$$
\begin{cases}V_{\ell, k}=e^{-\frac{i \pi(N-\ell) k}{2 N+m+1}}, \quad \ell=0, \ldots, m ; k=0, \ldots, 2 m+1, \\ V_{\ell, k}=e^{\frac{i \pi(N-\ell+m+1) k}{2 N+m+1}}, \quad \ell=m+1, \ldots, 2 m+1 ; k=0, \ldots, 2 m+1 .\end{cases}
$$

The solution of (11) can be derived explicitly through the inverse of the Vandermonde matrix (12). Let

$$
\alpha_{k}=e^{-\frac{i \pi(N-k)}{2 N+m+1}}, \beta_{k}=e^{\frac{i \pi(N-k)}{2 N+m+1}} .
$$

The standard technique for the calculation of the inverse (see details in 81 for a similar problem) implies

$$
\left\{\begin{array}{c}
V_{k, \ell}^{-1}=-\frac{\sum_{j=0}^{k} \gamma_{j} \alpha_{\ell}^{j}}{\alpha_{\ell}^{k+1} \prod_{i=0}^{m}\left(\alpha_{\ell}-\beta_{i}\right) \prod_{\substack{i=0 \\
i \neq \ell}}^{m}\left(\alpha_{\ell}-\alpha_{i}\right)}, \\
\ell=0, \ldots, m ; k=0, \ldots, 2 m+1, \\
V_{k, \ell}^{-1}=-\frac{\sum_{j=0}^{k} \gamma_{j} \beta_{\ell-m-1}^{j}}{\beta_{\ell-m-1}^{k+1} \prod_{\substack{i=0 \\
i \neq \ell-m-1}}^{m}\left(\beta_{\ell-m-1}-\beta_{i}\right) \prod_{i=0}^{m}\left(\beta_{\ell-m-1}-\alpha_{i}\right)}, \\
\ell=m+1, \ldots, 2 m+1 ; k=0, \ldots, 2 m+1,
\end{array}\right.
$$


where $\gamma_{j}$ are the coefficients of the following polynomial

$$
\prod_{i=0}^{m}\left(x-\alpha_{i}\right) \prod_{i=0}^{m}\left(x-\beta_{i}\right)=\sum_{j=0}^{2 m+2} \gamma_{j} x^{j} .
$$

Now, we get the following explicit form of the coefficients $F_{n, m}$ in the terms of the inverse $\left\{V_{k, \ell}^{-1}\right\}$

$$
\begin{aligned}
F_{n, m}=f_{n, m}-\sum_{k=0}^{2 m+1} e^{-\frac{i \pi n\left(2 N+k+\frac{1}{2}\right)}{2 N+m+1}} & \\
& \times\left(\sum_{\ell=0}^{m} V_{k, \ell}^{-1} e^{\frac{i \pi(N-\ell)\left(2 N+\frac{1}{2}\right)}{2 N+m+1}} f_{N-\ell, m}\right. \\
& \left.\quad+\sum_{\ell=0}^{m} V_{k, \ell+m+1}^{-1} e^{-\frac{i \pi(N-\ell)\left(2 N+\frac{1}{2}\right)}{2 N+m+1}} f_{-N+\ell, m}\right) .
\end{aligned}
$$

Algorithm $\mathrm{C}$ has several benefits. First and foremost, it provides the existence and uniqueness of the solution to the corresponding system of linear equations (see (10)). Second, practical solution can be achieved also by using the well-known Björk-Pereyra algorithm [120 for the solution to systems with Vandermonde matrices. This $O\left((2 m+2)^{2}\right)$ algorithm has a number of beneficial properties. In particular, under certain mild hypotheses [121], the magnitude of the numerical errors depends only on the machine precision used, and is independent of condition numbers of matrices. However, for the theoretical purposes, we will use the explicit solution.

\section{Preliminaries}

We consider some preliminary statements and lemmas for the main theorems.

Consider a sequence of complex numbers $\left\{y_{s}\right\}_{s=-\infty}^{\infty}$ and denote

$$
\hat{y}=\left\{y_{s}\right\}_{s=-\infty}^{\infty} \text {. }
$$

Let

$$
\begin{gathered}
\delta_{n}^{0}(\hat{y})=y_{n}, \\
\delta_{n}^{p}(\hat{y})=\delta_{n+1}^{p-1}(\hat{y})+2 \delta_{n}^{p-1}(\hat{y})+\delta_{n-1}^{p-1}(\hat{y}), p \geq 1,
\end{gathered}
$$

and

$$
\begin{gathered}
\Delta_{n}^{0}(\hat{y})=y_{n} \\
\Delta_{n}^{p}(\hat{y})=\Delta_{n}^{p-1}(\hat{y})+\Delta_{n-1}^{p-1}(\hat{y}), p \geq 1 .
\end{gathered}
$$


It is easy to verify that

$$
\delta_{n}^{p}(\hat{y})=\Delta_{n+p}^{2 p}(\hat{y})
$$

and

$$
\Delta_{n}^{p}(\hat{y})=\sum_{k=0}^{p}\left(\begin{array}{l}
p \\
k
\end{array}\right) y_{n-k}
$$

Consequently,

$$
\delta_{n}^{p}(\hat{y})=\sum_{k=0}^{2 p}\left(\begin{array}{c}
2 p \\
k
\end{array}\right) y_{n+p-k}
$$

Lemma 1 [91] Let

$$
\hat{u}=\left\{(-1)^{s} e^{\frac{i \pi \beta s}{2 N+m+1}}\right\}_{s=-\infty}^{\infty}, \beta \in \mathbb{R} .
$$

Then, for any $n$, the following estimate holds as $N \rightarrow \infty$

$$
\delta_{n}^{p}(\hat{u})=\frac{(-1)^{n}(\pi \beta)^{2 p}}{(2 N+m+1)^{2 p}} e^{\frac{i \pi \beta n}{2 N+m+1}}+O\left(N^{-2 p-1}\right) .
$$

Lemma 2 Let

$$
\left\{\begin{array}{l}
B_{n}(j)=\frac{(-1)^{n}}{(i \pi n)^{j+1}} e^{\frac{i \pi \beta n}{2 N+m+1}}, n \neq 0, \quad j \geq 0, \beta \in \mathbb{R}, \\
B_{0}(j)=0,
\end{array}\right.
$$

and

$$
\hat{v}_{j}=\left\{B_{n}(j)\right\}_{n=-\infty}^{\infty} .
$$

Then, the following estimate holds for $|n| \geq N$ as $N \rightarrow \infty$

$$
\delta_{n}^{p}\left(\hat{v}_{j}\right)=\frac{(-1)^{n+p}}{(i \pi n)^{j+1}} e^{\frac{i \pi \beta n}{2 N+m+1}} \sum_{t=2 p}^{\infty} \frac{\omega_{2 p, t}(-i \pi \beta)^{t}}{t !(2 N+m+1)^{t}}+O\left(n^{-j-2} N^{-2 p+1}\right),
$$

where

$$
\omega_{p, t}=\sum_{k=0}^{p}\left(\begin{array}{l}
p \\
k
\end{array}\right)(-1)^{k} k^{t}
$$

Proof. We have

$$
\begin{aligned}
\Delta_{n}^{p}\left(\hat{v}_{j}\right)=\frac{(-1)^{n} e^{\frac{i \pi \beta n}{2 N+m+1}}}{(i \pi n)^{j+1}} \sum_{k=0}^{p}\left(\begin{array}{l}
p \\
k
\end{array}\right) \frac{(-1)^{k} e^{-\frac{i \pi \beta k}{2 N+m+1}}}{\left(1-\frac{k}{n}\right)^{j+1}} \\
=\frac{(-1)^{n} e^{\frac{i \pi \beta n}{2 N+m+1}}}{(i \pi n)^{j+1}} \sum_{t=0}^{\infty} \omega_{p, t} \sum_{\tau=0}^{t}\left(\begin{array}{c}
\tau+j \\
\tau
\end{array}\right) \frac{(-i \pi \beta)^{t-\tau}}{(t-\tau) !(2 N+m+1)^{t-\tau}} \frac{1}{n^{\tau}} \\
=\frac{(-1)^{n} e^{\frac{i \pi \beta n}{2 N+m+1}}}{(i \pi n)^{j+1}} \sum_{t=p}^{\infty} \frac{\omega_{p, t}(-i \pi \beta)^{t}}{t !(2 N+m+1)^{t}}+O\left(n^{-j-2} N^{-p+1}\right) .
\end{aligned}
$$

Relation $\delta_{n}^{p}\left(\hat{v}_{j}\right)=\Delta_{n+p}^{2 p}\left(\hat{v}_{j}\right)$ completes the proof. 
Denote by $A C[-1,1]$ the set of all absolutely continuous functions on $[-1,1]$.

Lemma 3 Let $f^{(q+v)} \in A C[-1,1]$ for some $q, v \geq 0$ and

$$
f^{(k)}(-1)=f^{(k)}(1)=0, k=0, \ldots, q-1 .
$$

Then, for any $N$, the following estimate holds as $n \rightarrow \infty$

$$
f_{n, m}=(-1)^{n+1} \frac{\sigma}{2} \sum_{j=q}^{q+v} \frac{1}{N^{j+1}} \mu_{j, m}\left(f, \frac{n}{2 N+m+1}\right)+o\left(n^{-q-v-1}\right),
$$

where

$$
\mu_{j, m}(f, x)=\frac{f^{(j)}(1) e^{i \pi(m+1) x}-f^{(j)}(-1) e^{-i \pi(m+1) x}}{(2 i \pi x)^{j+1}} .
$$

Proof. The proof is straightforward by means of integration by parts.

The next lemma uses two-point Taylor expansions.

Theorem 1 [122] Let $f(z)$ be an analytic function on an open set $\Omega \subset$ $\mathbb{C}$ and $z_{1}, z_{2} \in \Omega$ with $z_{1} \neq z_{2}$. Then, $f(z)$ admits the two-point Taylor expansion

$$
\begin{array}{r}
f(z)=\sum_{n=0}^{N-1}\left[a_{n}\left(z_{1}, z_{2}\right)\left(z-z_{1}\right)+a_{n}\left(z_{2}, z_{1}\right)\left(z-z_{2}\right)\right]\left(z-z_{1}\right)^{n}\left(z-z_{2}\right)^{n} \\
+r_{N}\left(z_{1}, z_{2} ; z\right),
\end{array}
$$

where the coefficients $a_{n}\left(z_{1}, z_{2}\right)$ and $a_{n}\left(z_{2}, z_{1}\right)$ are given by Cauchy integral

$$
a_{n}\left(z_{1}, z_{2}\right) \equiv \frac{1}{2 \pi i\left(z_{2}-z_{1}\right)} \int_{\mathcal{C}} \frac{f(\omega) d \omega}{\left(\omega-z_{1}\right)^{n}\left(\omega-z_{2}\right)^{n+1}} .
$$

The remainder term $r_{N}\left(z_{1}, z_{2} ; z\right)$ is given by the Cauchy integral

$$
r_{N}\left(z_{1}, z_{2} ; z\right) \equiv \frac{1}{2 \pi i} \int_{\mathcal{C}} \frac{f(\omega) d \omega}{\left(\omega-z_{1}\right)^{N}\left(\omega-z_{2}\right)^{N}(\omega-z)}\left(z-z_{1}\right)^{N}(z-z 2)^{N} .
$$

The contour of integration $\mathcal{C}$ is a simple closed loop which encircles the points $z_{1}$ and $z_{2}\left(\right.$ for $\left.a_{n}\right)$ and $z, z_{1}$ and $z_{2}\left(\right.$ for $\left.r_{N}\right)$ in the counterclockwise direction and is contained in $\Omega$. The expansion (17) is convergent for $z$ inside the Cassini oval

$$
O_{z_{1}, z_{2}} \equiv\left\{z \in \Omega,\left|\left(z-z_{1}\right)\left(z-z_{2}\right)\right|<r\right\}
$$

where

$$
r \equiv \operatorname{In} f_{\omega \in \mathcal{C} \backslash \Omega}\left\{\left|\left(\omega-z_{1}\right)\left(\omega-z_{2}\right)\right|\right\} .
$$


Representation (18) is inappropriate for numerical computations. A more practical formula to compute the coefficients of the above two-point Taylor expansion is given in the following proposition.

Proposition 2 [122] Coefficients $a_{n}\left(z_{1}, z_{2}\right)$ in expansion (17) are also given by the formulas:

$$
a_{0}\left(z_{1}, z_{2}\right)=\frac{f\left(z_{2}\right)}{z_{2}-z_{1}}
$$

and, for $n=1,2,3, \ldots$,

$$
a_{n}\left(z_{1}, z_{2}\right)=\sum_{k=0}^{n} \frac{(n+k-1) !}{k !(n-k) !} \frac{(-1)^{n+1} n f^{(n-k)}\left(z_{2}\right)+(-1)^{k} k f^{(n-k)}\left(z_{1}\right)}{n !\left(z_{1}-z_{2}\right)^{n+k+1}} .
$$

Let

$$
\Phi_{j}(z)=\frac{1}{z^{\frac{1}{2}}(\ln z)^{j+1}}, \Psi_{j}(z)=\frac{z^{2 m+\frac{3}{2}}}{(\ln z)^{j+1}}, j \geq 0 .
$$

We denote by $\Phi_{j, p}(z)$ the $(2 p-1)$-th order two-point Taylor expansion of $\Phi_{j}(z)$ at the points $z=z_{1}$ and $z=z_{2}$

$$
\Phi_{j, p}(z)=\sum_{n=0}^{p-1}\left(\phi_{j}^{(n)}\left(z_{1}, z_{2}\right)\left(z-z_{1}\right)+\phi_{j}^{(n)}\left(z_{2}, z_{1}\right)\left(z-z_{2}\right)\right)\left(z-z_{1}\right)^{n}\left(z-z_{2}\right)^{n}
$$

where

$$
\phi_{j}^{(0)}\left(z_{1}, z_{2}\right)=\frac{\Phi_{j}\left(z_{2}\right)}{z_{2}-z_{1}}
$$

and

$\phi_{j}^{(n)}\left(z_{1}, z_{2}\right)=\sum_{k=0}^{n} \frac{(n+k-1) !}{k !(n-k) !} \frac{(-1)^{n+1} n \Phi_{j}^{(n-k)}\left(z_{2}\right)+(-1)^{k} k \Phi_{j}^{(n-k)}\left(z_{1}\right)}{n !\left(z_{1}-z_{2}\right)^{n+k+1}}, n>0$.

Then, the remainder $r_{p}\left(\Phi_{j}, z\right)$ of the corresponding Taylor expansion can be written as

$$
r_{p}\left(\Phi_{j}, z\right)=\Phi_{j}(z)-\Phi_{j, p}(z)=O\left(\left(z-z_{k}\right)^{p}\right), z \rightarrow z_{k}, k=1,2 .
$$

We use similar notations for $\Psi_{j}(z)$ with $\Psi_{j, m-1}(z), \psi_{j}^{(n)}\left(z_{1}, z_{2}\right)$ and $r_{p}\left(\Psi_{j}, z\right)$.

Lemma 4 Let $f^{(q+v+m)} \in A C[-1,1]$ for some $q, v, m \geq 0$ and

$$
f^{(k)}(-1)=f^{(k)}(1)=0, k=0, \ldots, q-1 .
$$

Then, the following estimate holds for $|n| \leq N+c(c \in \mathbb{N}$ is a constant)

$$
\begin{aligned}
F_{n, m}-f_{n, m} & =\frac{\sigma}{2} e^{\frac{i \pi n\left(m+\frac{1}{2}\right)}{2 N+m+1}} \sum_{j=q}^{q+v} \frac{(-1)^{n+j+1}}{2^{j+1} N^{j+1}} \\
& \times\left(f^{(j)}(1) Y_{n}\left(\Phi_{j}\right)-f^{(j)}(-1) Y_{n}\left(\Psi_{j}\right)\right)+o\left(N^{-q-v-1}\right), N \rightarrow \infty,
\end{aligned}
$$


where

$$
Y_{n}\left(\varphi_{j}\right)=\varphi_{j, m+1}\left(e^{-\frac{i \pi n}{2 N+m+1}}\right)+\sum_{\ell=0}^{2 m+1} e^{-\frac{i \pi n \ell}{2 N+m+1}} W_{\ell}\left(\varphi_{j}\right)
$$

and

$$
W_{\ell}\left(\varphi_{j}\right)=\sum_{k=0}^{m} V_{\ell, k}^{-1} r_{m+1}\left(\varphi_{j}, \alpha_{k}\right)+\sum_{k=0}^{m} V_{\ell, k+m+1}^{-1} r_{m+1}\left(\varphi_{j}, \beta_{k}\right) .
$$

Proof. Equation (14) implies

$$
\begin{aligned}
F_{n, m}-f_{n, m}= & -\sum_{\ell=0}^{2 m+1} e^{-\frac{i \pi n\left(2 N+\ell+\frac{1}{2}\right)}{2 N+m+1}} \\
& \times\left(\sum_{k=0}^{m} V_{\ell, k}^{-1} \beta_{k}^{2 N+\frac{1}{2}} f_{N-k, m}+\sum_{k=0}^{m} V_{\ell, k+m+1}^{-1} \alpha_{k}^{2 N+\frac{1}{2}} f_{-N+k, m}\right) .
\end{aligned}
$$

According to Lemma 3, we have

$f_{N-k, m}=(-1)^{N+k+1} \frac{\sigma}{2} \sum_{j=q}^{q+v+m} \frac{f^{(j)}(1) \beta_{k}^{m+1}-f^{(j)}(-1) \alpha_{k}^{m+1}}{(i \pi \sigma(N-k))^{j+1}}+o\left(N^{-q-v-m-1}\right)$,

and

$f_{-N+k, m}=(-1)^{N+k+1} \frac{\sigma}{2} \sum_{j=q}^{q+v+m} \frac{f^{(j)}(1) \alpha_{k}^{m+1}-f^{(j)}(-1) \beta_{k}^{m+1}}{(-i \pi \sigma(N-k))^{j+1}}+o\left(N^{-q-v-m-1}\right)$.

Taking into account that $V_{\ell, k}^{-1}=O\left(N^{m}\right)$, we get the following estimate

$$
F_{n, m}-f_{n, m}=I_{1}+I_{2}+o\left(N^{-q-v-1}\right), N \rightarrow \infty,
$$

where

$$
I_{1}=\frac{\sigma}{2} e^{-\frac{i \pi n\left(2 N+\frac{1}{2}\right)}{2 N+m+1}} \sum_{j=q}^{q+v+m} \frac{(-1)^{j+1} f^{(j)}(1)}{(2 N)^{j+1}} Z_{n}\left(\Phi_{j}\right)
$$

and

$$
I_{2}=-\frac{\sigma}{2} e^{-\frac{i \pi n\left(2 N+\frac{1}{2}\right)}{2 N+m+1}} \sum_{j=q}^{q+v+m} \frac{(-1)^{j+1} f^{(j)}(-1)}{(2 N)^{j+1}} Z_{n}\left(\Psi_{j}\right)
$$

with

$$
Z_{n}\left(\varphi_{j}\right)=\sum_{\ell=0}^{2 m+1} e^{-\frac{i \pi n \ell}{2 N+m+1}}\left(\sum_{k=0}^{m} V_{\ell, k}^{-1} \varphi_{j}\left(\alpha_{k}\right)+\sum_{k=0}^{m} V_{\ell, k+m+1}^{-1} \varphi_{j}\left(\beta_{k}\right)\right) .
$$

This completes the proof in view of the two-point Taylor expansions for functions $\Phi_{j}$ and $\Psi_{j}$ at the points $-\imath$ and $\imath$. 
Lemma 5 Let $f^{(q+2 m+1)} \in A C[-1,1]$ for some $q, m \geq 0$ and

$$
f^{(k)}(-1)=f^{(k)}(1)=0, k=0, \ldots, q-1 .
$$

Then, the following estimates hold for $p \in \mathbb{Z}$

$$
F_{N+p, m}=\frac{(-1)^{N+p+m}}{N^{q+m+2}}\left(\begin{array}{l}
m+p \\
m+1
\end{array}\right) C_{q, m}(f)+o\left(N^{-q-m-2}\right), N \rightarrow \infty,
$$

and

$$
F_{-N-p, m}=\frac{(-1)^{N+p+m}}{N^{q+m+2}}\left(\begin{array}{l}
m+p \\
m+1
\end{array}\right) \overline{C_{q, m}(f)}+o\left(N^{-q-m-2}\right), N \rightarrow \infty,
$$

where

$$
\begin{aligned}
& C_{q, m}(f)=\frac{(-1)^{q} \sqrt{\imath} \pi^{m+1}(m+1) !}{2^{q+1}} \\
& \quad \times\left(f^{(q)}(1) \phi_{q}^{(m+1)}(\imath,-\imath)-f^{(q)}(-1) \psi_{q}^{(m+1)}(\imath,-\imath)\right) .
\end{aligned}
$$

Proof. Lemma 3, with $v=2 m+1$ and Equation (14), implies

$$
\begin{gathered}
F_{N+p, m}=\frac{\sigma}{2} \sum_{\ell=0}^{2 m+1} e^{-\frac{i \pi(N+p)\left(2 N+\ell+\frac{1}{2}\right)}{2 N+m+1}}\left(\sum_{k=0}^{m} V_{\ell, k}^{-1} h_{k}(f)+\sum_{k=0}^{m} V_{\ell, k+m+1}^{-1} \overline{h_{k}(f)}\right) \\
-\frac{\sigma}{2} e^{-\frac{i \pi(N+p)\left(2 N+\frac{1}{2}\right)}{2 N+m+1}} h_{-p}(f)+o\left(N^{-q-m-2}\right)=T_{1}+T_{2}+o\left(N^{-q-m-2}\right),
\end{gathered}
$$

where

$$
\begin{aligned}
h_{k}(f)=e^{\frac{i \pi(N-k)\left(2 N+\frac{1}{2}\right)}{2 N+m+1}} \sum_{t=q}^{q+2 m+1} \frac{f^{(t)}(1) \alpha_{k}^{2 N}-f^{(t)}(-1) \beta_{k}^{2 N}}{(i \pi \sigma)^{t+1}(N-k)^{t+1}} \\
=\sum_{t=q}^{q+2 m+1} \frac{(-1)^{t+1}}{(2 N)^{t+1}}\left(f^{(t)}(1) \Phi_{t}\left(\alpha_{k}\right)-f^{(t)}(-1) \Psi_{t}\left(\alpha_{k}\right)\right),
\end{aligned}
$$

with

$$
T_{1}=-\frac{\sigma}{2} e^{-\frac{i \pi(N+p)\left(2 N+\frac{1}{2}\right)}{2 N+m+1}} \sum_{t=q}^{q+2 m+1} \frac{(-1)^{t+1}}{(2 N)^{t+1}} f^{(t)}(1)\left(\Phi_{t}\left(\alpha_{-p}\right)-Z_{N+p}\left(\Phi_{t}\right)\right),
$$

and

$$
T_{2}=\frac{\sigma}{2} e^{-\frac{i \pi(N+p)\left(2 N+\frac{1}{2}\right)}{2 N+m+1}} \sum_{t=q}^{q+2 m+1} \frac{(-1)^{t+1}}{(2 N)^{t+1}} f^{(t)}(-1)\left(\Psi_{t}\left(\alpha_{-p}\right)-Z_{N+p}\left(\Psi_{t}\right)\right) .
$$


First, we estimate $T_{1}$

$$
\begin{aligned}
& \Phi_{t}\left(\alpha_{-p}\right)-Z_{N+p}\left(\Phi_{t}\right)=\Phi_{t}\left(\alpha_{-p}\right)-\sum_{k=0}^{m} \sum_{\ell=0}^{2 m+1} \alpha_{-p}^{\ell} V_{\ell, k}^{-1} \Phi_{t}\left(\alpha_{k}\right) \\
&-\sum_{k=0}^{m} \sum_{\ell=0}^{2 m+1} \alpha_{-p}^{\ell} V_{\ell, k+m+1}^{-1} \Phi_{t}\left(\beta_{k}\right)=\sum_{k=0}^{m} \operatorname{res} \frac{\omega\left(\alpha_{-p}\right) \Phi_{t}(z)}{\omega(z)\left(z-\alpha_{-p}\right)} \\
&+\sum_{k=0}^{m} \operatorname{res}_{z=\beta_{k}} \frac{\omega\left(\alpha_{-p}\right) \Phi_{t}(z)}{\omega(z)\left(z-\alpha_{-p}\right)}+\underset{z=\alpha-p}{\operatorname{res}} \frac{\omega\left(\alpha_{-p}\right) \Phi_{t}(z)}{\omega(z)\left(z-\alpha_{-p}\right)},
\end{aligned}
$$

where

$$
\omega(z)=\prod_{k=0}^{m}\left(z-\alpha_{k}\right) \prod_{k=0}^{m}\left(z-\beta_{k}\right) .
$$

Hence,

$$
\Phi_{t}\left(\alpha_{-p}\right)-Z_{N+p}\left(\Phi_{t}\right)=\frac{1}{2 i \pi} \int_{\Gamma} \frac{\omega\left(\alpha_{-p}\right) \Phi_{t}(z)}{\omega(z)\left(z-\alpha_{-p}\right)} d z,
$$

where $\Gamma$ contains the points $\left\{\alpha_{k}\right\}_{k=0}^{m},\left\{\beta_{k}\right\}_{k=0}^{m}$ and $\alpha_{-p}$. We have

$$
\begin{aligned}
\Phi_{t}\left(\alpha_{-p}\right)-Z_{N+p}\left(\Phi_{t}\right)= & \frac{(i)^{m+1} \pi^{m+1}}{2 i \pi N^{m+1}} \prod_{k=0}^{m}(p+k) \int_{\Gamma} \frac{\Phi_{t}(z)}{(z+i)^{m+2}(z-i)^{m+1}} d z \\
\quad+O\left(N^{-m-2}\right) & \\
= & \frac{2 \imath^{m} \pi^{m+1}}{N^{m+1}} \phi_{t}^{(m+1)}(\imath,-\imath) \prod_{k=0}^{m}(p+k)+O\left(N^{-m-2}\right)
\end{aligned}
$$

which leads to the following estimate for $T_{1}$ according to the definition of the binomial coefficients $\left(\begin{array}{l}p \\ k\end{array}\right)$ for all $p \in \mathbb{Z}$ (see 123$]$ )

$$
\begin{aligned}
T_{1}=(-1)^{N+p+q+m} \frac{\sqrt{\imath} f^{(q)}(1) \pi^{m+1}(m+1) !}{2^{q+1} N^{q+m+2}}\left(\begin{array}{c}
m+p \\
m+1
\end{array}\right) \phi_{q}^{(m+1)}(\imath,-\imath) \\
+O\left(N^{-q-m-3}\right) .
\end{aligned}
$$

Similarly,

$$
\begin{array}{r}
T_{2}=-(-1)^{N+p+q+m} \frac{\sqrt{\imath} f^{(q)}(-1) \pi^{m+1}(m+1) !}{2^{q+1} N^{q+m+2}}\left(\begin{array}{c}
m+p \\
m+1
\end{array}\right) \psi_{q}^{(m+1)}(\imath,-\imath) \\
+O\left(N^{-q-m-3}\right) .
\end{array}
$$

Equations (32) and (33), along with (30), yield

$$
\begin{aligned}
F_{N+p}=\left(f^{(q)}(1) \phi_{q}^{(m+1)}(\imath,-\imath)-f^{(q)}(-1) \psi_{q}^{(m+1)}(\imath,-\imath)\right) \\
(-1)^{N+p+q+m} \frac{\sqrt{\imath} \pi^{m+1}(m+1) !}{2^{q+1} N^{q+m+2}}\left(\begin{array}{c}
m+p \\
m+1
\end{array}\right)+o\left(N^{-q-m-2}\right),
\end{aligned}
$$

which completes the proof of (27).

Relation $F_{-N-p}=\overline{F_{N+p}}$ completes the proof of $(28)$. 


\section{The Pointwise Convergence}

The main results of this section are Theorems 3 and 4 . They explore the pointwise convergence of the QP-approximations and reveal the exact constants of the corresponding asymptotic errors.

Let

$$
R_{N, m}(f, x)=f(x)-S_{N, m}(f, x) .
$$

Theorem 3 Let $f^{(q+2 m+2)} \in A C[-1,1]$ for some $q, m \geq 0$ and

$$
f^{(k)}(-1)=f^{(k)}(1)=0, \quad k=0, \ldots, q-1 .
$$

Then, the following estimate holds for $|x|<1$

$$
R_{N, m}(f, x)=(-1)^{N+m+1} \frac{D_{N, m}(f, x)}{N^{q+m+2}}+o\left(N^{-q-m-2}\right), N \rightarrow \infty,
$$

where

$$
\begin{gathered}
D_{N, m}(f, x)=\left(C_{q, m}(f) e^{i \pi N \sigma x}+\overline{C_{q, m}(f)} e^{-i \pi N \sigma x}\right) \sum_{k=0}^{\dot{m}} \frac{(-1)^{k}\left(\begin{array}{c}
m-k+1 \\
k
\end{array}\right)}{2^{2 k+2} \cos ^{2 k+2} \frac{\pi \sigma x}{2}} \\
-\left(C_{q, m}(f) e^{i \pi(N+1) \sigma x}+\overline{C_{q, m}(f)} e^{-i \pi(N+1) \sigma x}\right) \sum_{k=0}^{\dot{m}-1} \frac{(-1)^{k}\left(\begin{array}{c}
m-k-1 \\
k
\end{array}\right)}{2^{2 k+4} \cos ^{2 k+4} \frac{\pi \sigma x}{2}}
\end{gathered}
$$

with $\dot{m}=\left[\frac{m+1}{2}\right]$ and $C_{q, m}(f)$ defined by 29 .

Proof. We extend $f$ outside of $[-1,1]$ by $f(x) \equiv 0$ and consider its representation by the Fourier series expansion on $[-1 / \sigma, 1 / \sigma]$ via quasi-periodic exponential functions. The function $f$ is sufficiently smooth on $(-1,1)$ for the pointwise convergence of the expansion

$$
f(x)=\sum_{n=-\infty}^{\infty} f_{n, m} e^{i \pi n \sigma x}, x \in(-1,1)
$$

We use it in (34) and write the error of the QP-approximation in the form

$$
R_{N, m}(f, x)=\sum_{n=-N}^{N}\left(f_{n, m}-F_{n, m}\right) e^{i \pi n \sigma x}+\sum_{|n|>N} f_{n, m} e^{i \pi n \sigma x}, \quad x \in(-1,1) .
$$


The application of the Abel transformation leads to the expansion

$$
\begin{gathered}
R_{N, m}(f, x)=\frac{F_{N+1, m} e^{i \pi N \sigma x}-F_{N, m} e^{i \pi(N+1) \sigma x}}{\left(1+e^{-i \pi \sigma x}\right)\left(1+e^{i \pi \sigma x}\right)} \\
+\frac{F_{-N-1, m} e^{-i \pi N \sigma x}-F_{-N, m} e^{-i \pi(N+1) \sigma x}}{\left(1+e^{-i \pi \sigma x}\right)\left(1+e^{i \pi \sigma x}\right)} \\
+\frac{1}{\left(1+e^{-i \pi \sigma x}\right)\left(1+e^{i \pi \sigma x}\right)} \sum_{n=-N}^{N} \delta_{n}^{1}\left(\left\{f_{s, m}-F_{s, m}\right\}\right) e^{i \pi \sigma n x} \\
\quad+\frac{1}{\left(1+e^{-i \pi \sigma x}\right)\left(1+e^{i \pi \sigma x}\right)} \sum_{|n|>N} \delta_{n}^{1}\left(\left\{f_{s, m}\right\}\right) e^{i \pi \sigma n x} .
\end{gathered}
$$

Reiteration of this transformation up to $m+1$ times implies

$$
R_{N, m}(f, x)=R_{1}-R_{2}+R_{3}-R_{4}+r
$$

where

$$
\begin{gathered}
R_{1}=e^{i \pi N \sigma x} \sum_{k=0}^{\dot{m}} \frac{\delta_{N+1}^{k}\left(\left\{F_{s, m}\right\}\right)}{\left(1+e^{-i \pi \sigma x}\right)^{k+1}\left(1+e^{i \pi \sigma x}\right)^{k+1}}, \\
R_{2}=e^{i \pi(N+1) \sigma x} \sum_{k=0}^{\dot{m}} \frac{\delta_{N}^{k}\left(\left\{F_{s, m}\right\}\right)}{\left(1+e^{-i \pi \sigma x}\right)^{k+1}\left(1+e^{i \pi \sigma x}\right)^{k+1}}, \\
R_{3}=e^{-i \pi N \sigma x} \sum_{k=0}^{\dot{m}} \frac{\delta_{-N-1}^{k}\left(\left\{F_{s, m}\right\}\right)}{\left(1+e^{-i \pi \sigma x}\right)^{k+1}\left(1+e^{i \pi \sigma x}\right)^{k+1}}, \\
R_{4}=e^{-i \pi(N+1) \sigma x} \sum_{k=0}^{\dot{m}} \frac{\delta_{-N}^{k}\left(\left\{F_{s, m}\right\}\right)}{\left(1+e^{-i \pi \sigma x}\right)^{k+1}\left(1+e^{i \pi \sigma x}\right)^{k+1}},
\end{gathered}
$$

and

$$
\begin{array}{r}
r=\frac{1}{\left(1+e^{-i \pi \sigma x}\right)^{\dot{m}+1}\left(1+e^{i \pi \sigma x}\right)^{\dot{m}+1}} \sum_{n=-N}^{N} \delta_{n}^{\dot{m}+1}\left(\left\{f_{s, m}-F_{s, m}\right\}\right) e^{i \pi \sigma n x} \\
+\frac{1}{\left(1+e^{-i \pi \sigma x}\right)^{\dot{m}+1}\left(1+e^{i \pi \sigma x}\right)^{\dot{m}+1}} \sum_{|n|>N} \delta_{n}^{\dot{m}+1}\left(\left\{f_{s, m}\right\}\right) e^{i \pi \sigma n x}
\end{array}
$$

We prove that

$$
r=o\left(N^{-q-m-2}\right), N \rightarrow \infty,|x|<1 .
$$

The application of similar transformation to (35) leads to the following expansion

$$
r=r_{1}+r_{2}+r_{3}+r_{4}
$$


where

$$
\begin{gathered}
r_{1}=\frac{\delta_{-N-1}^{\dot{m}+1}\left(\left\{F_{s, m}\right\}\right) e^{-i \pi N \sigma x}-\delta_{N}^{\dot{m}+1}\left(\left\{F_{s, m}\right\}\right) e^{i \pi(N+1) \sigma x}}{\left(1+e^{-i \pi \sigma x}\right)^{\dot{m}+2}\left(1+e^{i \pi \sigma x}\right)^{\dot{m}+2}}, \\
r_{2}=\frac{\delta_{N+1}^{\dot{m}+1}\left(\left\{F_{s, m}\right\}\right) e^{i \pi N \sigma x}-\delta_{-N}^{\dot{m}+1}\left(\left\{F_{s, m}\right\}\right) e^{-i \pi(N+1) \sigma x}}{\left(1+e^{-i \pi \sigma x}\right)^{\dot{m}+2}\left(1+e^{i \pi \sigma x}\right)^{\dot{m}+2}}, \\
r_{3}=\frac{1}{\left(1+e^{-i \pi \sigma x}\right)^{\dot{m}+2}\left(1+e^{i \pi \sigma x}\right)^{m^{\prime}+2}} \sum_{n=-N}^{N} \delta_{n}^{\dot{m}+2}\left(\left\{f_{s}-F_{s, m}\right\}\right) e^{i \pi \sigma n x},
\end{gathered}
$$

and

$$
r_{4}=\frac{1}{\left(1+e^{-i \pi \sigma x}\right)^{\dot{m}+2}\left(1+e^{i \pi \sigma x}\right)^{\dot{m}^{\prime}+2}} \sum_{|n|>N} \delta_{n}^{\dot{m}+2}\left(\left\{f_{s}\right\}\right) e^{i \pi \sigma n x} .
$$

Equation (15) shows that

$$
\delta_{N}^{\dot{m}+1}\left(\left\{F_{s, m}\right\}\right)=\sum_{k=0}^{2 \dot{m}+2}\left(\begin{array}{c}
2 \dot{m}+2 \\
k
\end{array}\right) F_{N+\dot{m}+1-k, m} .
$$

In view of the following identity (see 123 )

$$
\sum_{k=0}^{2 \dot{m}+2}(-1)^{k}\left(\begin{array}{c}
2 \dot{m}+2 \\
k
\end{array}\right)\left(\begin{array}{c}
m+\dot{m}+1-k \\
m+1
\end{array}\right)=0
$$

Equation (36), and Lemma 5, we derive

$$
\begin{aligned}
\delta_{N}^{\dot{m}+1}\left(\left\{F_{s, m}\right\}\right)= & C_{q, m}(f) \frac{(-1)^{N+m+\dot{m}+1}}{N^{q+m+2}} \\
\times & \times \sum_{k=0}^{2 \dot{m}+2}(-1)^{k}\left(\begin{array}{c}
2 \dot{m}+2 \\
k
\end{array}\right)\left(\begin{array}{c}
m+\dot{m}+1-k \\
m+1
\end{array}\right) \\
& \quad+o\left(N^{-q-m-2}\right)=o\left(N^{-q-m-2}\right), N \rightarrow \infty .
\end{aligned}
$$

Similarly,

$$
\delta_{-N-1}^{\dot{m}+1}\left(\left\{F_{s, m}\right\}\right)=o\left(N^{-q-m-2}\right), N \rightarrow \infty .
$$

It means that

$$
r_{1}=o\left(N^{-q-m-2}\right) .
$$

By the same arguments, we get

$$
r_{2}=o\left(N^{-q-m-2}\right) \text {. }
$$

In view of Lemma 4 (with $v=m+2$ ) and Lemma 1, we have

$$
\delta_{n}^{\dot{m}+2}\left(\left\{F_{s, m}-f_{s, m}\right\}\right)=o\left(N^{-q-m-3}\right), N \rightarrow \infty .
$$


It shows that

$$
r_{3}=o\left(N^{-q-m-2}\right) .
$$

Lemma 3 (with $v=2 m+2$ ) indicates that

$$
\begin{aligned}
& f_{n, m}=\sum_{k=q}^{q+2 m+2} \frac{f^{(k)}(1)(-1)^{n+1} e^{\frac{i \pi n(m+1)}{2 N+m+1}}-f^{(k)}(-1)(-1)^{n+1} e^{-\frac{i \pi n(m+1)}{2 N+m+1}}}{2 \sigma^{k}(i \pi n)^{k+1}} \\
&+o\left(n^{-q-2 m-3}\right), n \rightarrow \infty .
\end{aligned}
$$

According to Lemma (2), we obtain

$$
\begin{gathered}
\delta_{n}^{\dot{m}+2}\left(\left\{f_{s, m}\right\}\right)=\frac{f^{(q)}(1)(-1)^{n+\dot{m}+1} e^{\frac{i \pi(m+1) n}{2 N+m+1}}}{(i \pi n)^{q+1}} \sum_{t=2 \dot{m}+4}^{\infty} \frac{\omega_{2 \dot{m}+4, t}(-i \pi)^{t}(m+1)^{t}}{t !(2 N+m+1)^{t}} \\
+\frac{f^{(q)}(-1)(-1)^{n+\dot{m}+1} e^{-\frac{i \pi(m+1) n}{2 N+m+1}}}{(i \pi n)^{q+1}} \sum_{t=2 \dot{m}+4}^{\infty} \frac{\omega_{2 \dot{m}+4, t}(i \pi)^{t}(m+1)^{t}}{t !(2 N+m+1)^{t}} \\
+o\left(n^{-q-2} N^{-2 \dot{m}-1}\right),|n|>N, N \rightarrow \infty .
\end{gathered}
$$

It means that

$$
r_{4}=o\left(N^{-q-m-2}\right) .
$$

Finally, we conclude that

$$
R_{N, m}(f, x)=R_{1}+R_{2}+R_{3}+R_{4}+o\left(N^{-q-m-2}\right), N \rightarrow \infty .
$$

We continue with estimates of $R_{1}, R_{2}, R_{3}$ and $R_{4}$.

Let us start with $R_{1}$. Equation (15) leads to the following equation

$$
\delta_{N+1}^{k}\left(\left\{F_{s, m}\right\}\right)=\sum_{s=0}^{2 k}\left(\begin{array}{c}
2 k \\
s
\end{array}\right) F_{N+1+k-s, m},
$$

and, therefore,

$$
\begin{aligned}
& R_{1}=e^{i \pi N \sigma x} \sum_{k=0}^{m} \frac{\delta_{N+1}^{k}\left(\left\{F_{s, m}\right\}\right)}{\left(1+e^{-i \pi \sigma x}\right)^{k+1}\left(1+e^{i \pi \sigma x}\right)^{k+1}} \\
& \quad=e^{i \pi N \sigma x} \sum_{k=0}^{\dot{m}} \frac{1}{2^{2 k+2} \cos ^{2 k+2} \frac{\pi \sigma x}{2}} \sum_{s=0}^{2 k}\left(\begin{array}{c}
2 k \\
s
\end{array}\right) F_{N+1+k-s, m} .
\end{aligned}
$$

Then, Lemma 5 implies

$$
\begin{aligned}
& R_{1}=C_{q, m}(f) \frac{(-1)^{N+m+1}}{N^{q+m+2}} e^{i \pi N \sigma x} \\
& \times \sum_{k=0}^{\dot{m}} \frac{(-1)^{k}}{2^{2 k+2} \cos ^{2 k+2} \frac{\pi \sigma x}{2}} \sum_{s=0}^{2 k}(-1)^{s}\left(\begin{array}{c}
2 k \\
s
\end{array}\right)\left(\begin{array}{c}
m+1+k-s \\
m+1
\end{array}\right)+o\left(N^{-q-m-2}\right) .
\end{aligned}
$$


The following identity (see 123)

$$
\sum_{s=0}^{2 k}(-1)^{s}\left(\begin{array}{c}
2 k \\
s
\end{array}\right)\left(\begin{array}{c}
m+1+k-s \\
m+1
\end{array}\right)=\left(\begin{array}{c}
m-k+1 \\
k
\end{array}\right)
$$

deduces

$$
\begin{aligned}
& R_{1}=C_{q, m}(f) \frac{(-1)^{N+m+1}}{N^{q+m+2}} e^{i \pi N \sigma x} \\
& \times \sum_{k=0}^{m}\left(\begin{array}{c}
m-k+1 \\
k
\end{array}\right) \frac{(-1)^{k}}{2^{2 k+2} \cos ^{2 k+2} \frac{\pi \sigma x}{2}}+o\left(N^{-q-m-2}\right) .
\end{aligned}
$$

Now, we estimate $R_{3}$. We have

$$
\delta_{-N-1}^{k}\left(\left\{F_{s, m}\right\}\right)=\sum_{s=0}^{2 k}\left(\begin{array}{c}
2 k \\
s
\end{array}\right) F_{-N-1+k-s, m}=\sum_{s=0}^{2 k}\left(\begin{array}{c}
2 k \\
s
\end{array}\right) F_{-N-1-k+s, m},
$$

and consequently,

$$
\begin{aligned}
R_{3}=e^{-i \pi N \sigma x} \sum_{k=0}^{\dot{m}} & \frac{\delta_{-N-1}^{k}\left(\left\{F_{s, m}\right\}\right)}{\left(1+e^{-i \pi \sigma x}\right)^{k+1}\left(1+e^{i \pi \sigma x}\right)^{k+1}} \\
& =e^{-i \pi N \sigma x} \sum_{k=0}^{\dot{m}} \frac{1}{2^{2 k+2} \cos ^{2 k+2} \frac{\pi \sigma x}{2}} \sum_{s=0}^{2 k}\left(\begin{array}{c}
2 k \\
s
\end{array}\right) F_{-N-1-k+s, m} .
\end{aligned}
$$

In view of Lemma 5 ,

$$
\begin{aligned}
& R_{3}=\overline{C_{q, m}(f)} \frac{(-1)^{N+m+1}}{N^{q+m+2}} e^{-i \pi N \sigma x} \\
& \quad \times \sum_{k=0}^{\dot{m}}\left(\begin{array}{c}
m-k+1 \\
k
\end{array}\right) \frac{(-1)^{k}}{2^{2 k+2} \cos ^{2 k+2} \frac{\pi \sigma x}{2}}+o\left(N^{-q-m-2}\right) .
\end{aligned}
$$

By summarizing, we get

$$
\begin{aligned}
R_{1}+R_{3}=\frac{(-1)^{N+m+1}}{N^{q+m+2}}\left(C_{q, m}(f) e^{i \pi N \sigma x}+\overline{C_{q, m}(f)} e^{-i \pi N \sigma x}\right) \\
\times \sum_{k=0}^{\dot{m}}\left(\begin{array}{c}
m-k+1 \\
k
\end{array}\right) \frac{(-1)^{k}}{2^{2 k+2} \cos ^{2 k+2} \frac{\pi \sigma x}{2}}+o\left(N^{-q-m-2}\right) .
\end{aligned}
$$

Similarly,

$$
\begin{aligned}
R_{2}+R_{4}=\frac{(-1)^{N+m+1}}{N^{q+m+2}}\left(C_{q, m}(f) e^{i \pi(N+1) \sigma x}+\overline{C_{q, m}(f)} e^{-i \pi(N+1) \sigma x}\right) \\
\times \sum_{k=0}^{\dot{m}-1}\left(\begin{array}{c}
m-k-1 \\
k
\end{array}\right) \frac{(-1)^{k}}{2^{2 k+4} \cos ^{2 k+4} \frac{\pi \sigma x}{2}}+o\left(N^{-q-m-2}\right),
\end{aligned}
$$

which completes the proof. 
Remark 1 The estimate of Theorem 3 is valid only for a fixed $x \neq \pm 1$ and is not a uniform estimate on $[-1,1]$ (see Theorem 4).

The next theorem reveals the convergence rate of the QP-approximations exactly at the points $x= \pm 1$. Preliminary, we need the special case of Lemma 4 with $v=0$.

Lemma 6 Let $f^{(q+m)} \in A C[-1,1]$ for some $q, m \geq 0$ and

$$
f^{(k)}(-1)=f^{(k)}(1)=0, k=0, \ldots, q-1 .
$$

Then, the following estimate holds for $|n| \leq N+c(c \in \mathbb{N}$ is a constant)

$$
F_{n, m}-f_{n, m}=\frac{(-1)^{n}}{N^{q+1}} \frac{\sigma}{2} \nu_{q, m}\left(f, \frac{n}{2 N+m+1}\right)+o\left(N^{-q-1}\right), N \rightarrow \infty,
$$

where

$$
\begin{aligned}
\nu_{q, m}(f, x)=\frac{(-1)^{q+1}}{2^{q+1}} e^{\frac{i \pi(2 m+1) x}{2}} & \\
& \times\left(f^{(q)}(1) \Phi_{q, m+1}\left(e^{-i \pi x}\right)-f^{(q)}(-1) \Psi_{q, m+1}\left(e^{-i \pi x}\right)\right) .
\end{aligned}
$$

Theorem 4 Let $f^{(q+m)} \in A C[-1,1]$ for some $q, m \geq 0$ and

$$
f^{(k)}(-1)=f^{(k)}(1)=0, \quad k=0, \ldots, q-1 .
$$

Then, the following estimate holds as $N \rightarrow \infty$

$$
\begin{aligned}
S_{N, m}(f, \pm 1)=\frac{f( \pm 1)}{2}+ & \frac{1}{N^{q}} \int_{-1 / 2}^{1 / 2} e^{\mp i \pi(m+1) x} \nu_{q, m}(f, x) d x \\
& +\frac{1}{N^{q}} \int_{|x|>1 / 2} e^{\mp i \pi(m+1) x} \mu_{q, m}(f, x) d x+o\left(N^{-q}\right),
\end{aligned}
$$

where $\mu_{q, m}(f, x)$ and $\nu_{q, m}(f, x)$ are defined by (16) and (38), respectively.

Proof. As in the proof of Theorem 3, we extend $f$ outside of $[-1,1]$ by $f(x) \equiv 0$ and consider its representation by the Fourier series expansion on $[-1 / \sigma, 1 / \sigma]$ via quasi-periodic exponential functions. Function $f$ is smooth on $(-1,1)$ but may have discontinuities at $x= \pm 1$ if $f( \pm 1) \neq 0$. It means that

$$
\frac{f( \pm 1)}{2}=\sum_{n=-\infty}^{\infty} f_{n, m} e^{ \pm i \pi n \sigma}
$$

and

$$
S_{N, m}(f, \pm 1)-\frac{f( \pm 1)}{2}=\sum_{n=-N}^{N}\left(F_{n, m}-f_{n, m}\right) e^{ \pm i \pi n \sigma}-\sum_{|n|>N} f_{n, m} e^{ \pm i \pi n \sigma}
$$


In view of Lemmas 3 and 6, we have

$$
\begin{aligned}
& \sum_{|n|>N} f_{n, m} e^{ \pm i \pi n \sigma}=-\frac{1}{N^{q+1}} \frac{\sigma}{2} \sum_{|n|>N} \mu_{q, m}\left(f, \frac{n}{2 N+m+1}\right) e^{\mp \frac{i \pi n(m+1)}{2 N+m+1}} \\
& +o\left(N^{-q}\right)=-\frac{1}{N^{q}} \int_{|x|>1 / 2} e^{\mp i \pi(m+1) x} \mu_{q, m}(f, x) d x+o\left(N^{-q}\right)
\end{aligned}
$$

and

$$
\begin{aligned}
\sum_{n=-N}^{N}\left(F_{n, m}-f_{n, m}\right) e^{ \pm i \pi n \sigma} & \\
=\frac{1}{N^{q+1}} \frac{\sigma}{2} \sum_{n=-N}^{N} \nu_{q, m} & \left(f, \frac{n}{2 N+m+1}\right) e^{\mp \frac{i \pi n(m+1)}{2 N+m+1}}+o\left(N^{-q}\right) \\
& =\frac{1}{N^{q}} \int_{-1 / 2}^{1 / 2} e^{\mp i \pi(m+1) x} \nu_{q, m}(f, x) d x+o\left(N^{-q}\right) .
\end{aligned}
$$

These conclude the proof.

Remark 2 Theorem 4 shows convergence rate $O\left(N^{-q}\right)$ as $N \rightarrow \infty$ at $x=$ \pm 1 . It means that the QP-approximations do not converge at the endpoints for $q=0$. Accelerated convergence can be achieved by the polynomial correction approach described in Section 5.

Let us compare the behaviors of the quasi-periodic approximations (Algorithm C) and interpolations (see 994,95]). The QP-approximation is written via Vandermonde matrix $(12)$ of size $(2 m+2) \times(2 m+2)$, where $(2 m+2)$ is the number of points outside of $[-1,1]$ used for extensions. Recall also that $m=-1$ corresponds to the classical truncated Fourier series (without the extension). Theorem 3 proves the convergence rate $O\left(N^{-q-m-2}\right)$. It means that for each pair of points participating in a function extension (per point for the left and right extensions), the QP-approximation gains additional $O\left(N^{-1}\right)$ factor in the convergence rate compared to the Krylov-Lanczos approach.

The QP-interpolation is realized by the following Vandermonde matrix

$$
\left\{e^{\frac{2 i \pi(\ell+N)(s-1)}{2 N+m+1}}\right\}_{\ell, s=1}^{m}
$$

of size $m \times m, m \geq 1$. It means that $m=1$ of the QP-interpolation corresponds to $m=0$ of the QP-approximation. The next theorem, proved in [91], revealed the convergence rate of the $\mathrm{QP}$-interpolation, where $i_{N, m}(f, x)$ corresponded to the error of the QP-interpolation. 
Theorem 5 g1] Let $f^{(q+2 m)} \in A C[-1,1]$ for some $m \geq 1, q \geq 0$ and

$$
f^{(k)}(-1)=f^{(k)}(1)=0, \quad k=0, \ldots, q-1 .
$$

Then, the following estimate holds for $|x|<1$

$$
i_{N, m}(f, x)=O\left(N^{-q-m-1}\right), N \rightarrow \infty .
$$

We see that each additional $m$ increases the convergence rate by the factor $O\left(N^{-1}\right)$. In this sense, the QP-interpolation is twice more effective compared to the QP-approximation.

\section{The Convergence in the $L_{2}$-norm}

Theorem 6 is the main result of this section. It reveals the behavior of the QP-approximation in the $L_{2}$-norm.

Theorem 6 Let $f^{(q+m)} \in A C[-1,1]$ for some $q, m \geq 0, q^{2}+m^{2} \neq 0$, and

$$
f^{(k)}(-1)=f^{(k)}(1)=0, k=0, \ldots, q-1 .
$$

Then, the following estimate holds

$$
\lim _{N \rightarrow \infty} N^{q+\frac{1}{2}}\left\|R_{N, m}(f, x)\right\|_{L_{2}(-1,1)}=c_{q, m}(f),
$$

where

$$
\begin{aligned}
c_{q, m}^{2}(f)=\int_{-1 / 2}^{1 / 2}\left|\nu_{q, m}(f, x)\right|^{2} d x & +\int_{|x|>1 / 2}\left|\mu_{q, m}(f, x)\right|^{2} d x \\
-\frac{m+1}{2} \int_{-1}^{1} \mid \int_{-1 / 2}^{1 / 2} \nu_{q, m}(f, h) e^{i \pi(m+1) x h} d h & \\
& +\left.\int_{|h|>1 / 2} \mu_{q, m}(f, h) e^{i \pi(m+1) x h} d h\right|^{2} d x
\end{aligned}
$$

and functions $\mu_{q, m}(f, x)$ and $\nu_{q, m}(f, x)$ are defined by (16) and (38), respectively.

Proof. We proceed as in the proof of Theorem 3 and extend $f$ outside of $[-1,1]$ by $f(x) \equiv 0$ :

$$
R_{N, m}(f, x)=\sum_{n=-N}^{N}\left(f_{n, m}-F_{n, m}\right) e^{i \pi n \sigma x}+\sum_{|n|>N} f_{n, m} e^{i \pi n \sigma x}=\sum_{n=-\infty}^{\infty} c_{n} e^{i \pi n \sigma x}
$$


where

$$
\left\{\begin{array}{l}
c_{n}=f_{n, m}-F_{n, m},|n| \leq N, \\
c_{n}=f_{n, m},|n|>N .
\end{array}\right.
$$

Then, we calculate the $L_{2}[-1,1]$-norm of the error:

$$
\begin{gathered}
\left\|R_{N, m}(f, x)\right\|_{L_{2}(-1,1)}^{2}=\int_{-1}^{1}\left|R_{N, m}(f, x)\right|^{2} d x=\int_{-1}^{1} \sum_{n, s=-\infty}^{\infty} c_{n} \bar{c}_{s} e^{i \pi(n-s) \sigma x} d x \\
=\int_{-\frac{1}{\sigma}}^{\frac{1}{\sigma}} \sum_{n, s=-\infty}^{\infty} c_{n} \bar{c}_{s} e^{i \pi(n-s) \sigma x} d x-\int_{-\frac{1}{\sigma}}^{-1} \sum_{n, s=-\infty}^{\infty} c_{n} \bar{c}_{s} e^{i \pi(n-s) \sigma x} d x \\
-\int_{1}^{\frac{1}{\sigma}} \sum_{n, s=-\infty}^{\infty} c_{n} \bar{c}_{s} e^{i \pi(n-s) \sigma x} d x=I_{1}-I_{2}-I_{3},
\end{gathered}
$$

where

$$
\begin{aligned}
& I_{1}=\int_{-\frac{1}{\sigma}}^{\frac{1}{\sigma}} \sum_{n, s=-\infty}^{\infty} c_{n} \bar{c}_{s} \cdot e^{i \pi(n-s) \sigma x} d x=\frac{2}{\sigma} \sum_{n=-\infty}^{\infty}\left|c_{n}\right|^{2} \\
&= \frac{2}{\sigma} \sum_{n=-N}^{N}\left|f_{n, m}-F_{n, m}\right|^{2}+\frac{2}{\sigma} \sum_{|n|>N}\left|f_{n, m}\right|^{2}, \\
& I_{2}=\int_{-\frac{1}{\sigma}}^{-1} \sum_{n, s=-\infty}^{\infty} c_{n} \bar{c}_{s} e^{i \pi(n-s) \sigma x} d x \\
&=\frac{m+1}{2 N} \int_{0}^{1} \sum_{n, s=-\infty}^{\infty}(-1)^{n+s} c_{n} \bar{c}_{s} e^{i \pi(n-s) \frac{m+1}{2 N+m+1} t} d t,
\end{aligned}
$$

and

$$
\begin{aligned}
I_{3}=\int_{1}^{\frac{1}{\sigma}} \sum_{n, s=-\infty}^{\infty} c_{n} \bar{c}_{s} e^{i \pi(n-s) \sigma x} d x & \\
& =\frac{m+1}{2 N} \int_{0}^{1} \sum_{n, s=-\infty}^{\infty}(-1)^{n+s} c_{n} \bar{c}_{s} e^{-i \pi(n-s) \frac{m+1}{2 N+m+1} t} d t .
\end{aligned}
$$

First, we estimate $I_{1}$. Lemma 3 and Remark 6 lead to the estimate

$$
\begin{aligned}
I_{1}= & \frac{1}{(2 N+m+1) N^{2 q+1}} \sum_{n=-N}^{N}\left|\nu_{q, m}\left(f, \frac{n}{2 N+m+1}\right)\right|^{2} \\
& +\frac{1}{(2 N+m+1) N^{2 q+1}} \sum_{|n|>N}\left|\mu_{q, m}\left(f, \frac{n}{2 N+m+1}\right)\right|^{2}+o\left(N^{-2 q-1}\right) .
\end{aligned}
$$


By tending $N$ to infinity and replacing the sums by the corresponding integrals, we get

$$
\lim _{N \rightarrow \infty} N^{2 q+1} I_{1}=\int_{-1 / 2}^{1 / 2}\left|\nu_{q, m}(f, x)\right|^{2} d x+\int_{|x|>1 / 2}\left|\mu_{q, m}(f, x)\right|^{2} d x .
$$

Second, we estimate $I_{2}$. We rewrite it as follows

$$
I_{2}=\frac{m+1}{2 N} \int_{0}^{1}|S(t)|^{2} d t
$$

where

$$
\begin{aligned}
S(t)=\sum_{n=-\infty}^{\infty}(-1)^{n} c_{n} e^{i \pi(m+1) t \frac{n}{2 N+m+1}} & \\
=\sum_{n=-N}^{N}(-1)^{n}\left(f_{n, m}-F_{n, m}\right) e^{i \pi(m+1) t \frac{n}{2 N+m+1}} & +\sum_{|n|>N}(-1)^{n} f_{n, m} e^{i \pi(m+1) t \frac{n}{2 N+m+1}}
\end{aligned}
$$

According to Lemma 3 and Remark 6, we obtain

$$
\begin{gathered}
S(t)=-\frac{1}{(2 N+m+1) N^{q}} \sum_{n=-N}^{N} \nu_{q, m}\left(f, \frac{n}{2 N+m+1}\right) e^{i \pi(m+1) t \frac{n}{2 N+m+1}} \\
-\frac{1}{(2 N+m+1) N^{q}} \sum_{|n|>N} \mu_{q, m}\left(f, \frac{n}{2 N+m+1}\right) e^{i \pi(m+1) t \frac{n}{2 N+m+1}}+o\left(N^{-q}\right) .
\end{gathered}
$$

By tending $N$ to infinity and replacing the sums by the corresponding integrals, we derive

$$
\begin{aligned}
\lim _{N \rightarrow \infty} N^{q} S(t)=-\int_{-1 / 2}^{1 / 2} \nu_{q, m}(f, h) e^{i \pi(m+1) t h} d h & \\
& -\int_{|h|>1 / 2} \mu_{q, m}(f, h) e^{i \pi(m+1) t h} d h .
\end{aligned}
$$

Hence,

$$
\begin{aligned}
\lim _{N \rightarrow \infty} N^{2 q+1} I_{2}=\frac{m+1}{2} \int_{0}^{1} \mid \int_{-1 / 2}^{1 / 2} \nu_{q, m}(f, h) e^{i \pi(m+1) t h} d h \\
\quad+\left.\int_{|h|>1 / 2} \mu_{q, m}(f, h) e^{i \pi(m+1) t h} d h\right|^{2} d t .
\end{aligned}
$$

We similarly estimate $I_{3}$ and complete the proof. 
Theorem 6 is not valid for $q=m=0$. However, the same estimate can be proved for $q=m=0$ if $f^{\prime} \in L_{2}[-1,1]$. The proof is identical to the one of Theorem 6 and is omitted.

We see that the convergence rate of the QP-approximation in the $L_{2^{-}}$ norm is identical to the rates of the truncated Fourier series and QP-interpolation (see [93]). The constants of the asymptotic errors are different. Formally, $c_{q,-1}(f)$ corresponds to the classical Fourier series. Note that the last negative term in (40) vanishes for $m=-1$. When $m \neq-1$, the negative term reduces the $L_{2}$-constant corresponding to the QP-approximation compared to the classical Fourier series.

Unfortunately, the rate of descent depends on the approximated function. Our experiments also reveal that the constant is decreasing as $m$ is increasing. However, the proof of this observation is still unspecified. Let us show it for the following elementary function

$$
f(x)=\left(x^{2}-1\right)^{q} \sin (x-1), q=0,1,2, \cdots .
$$

Table 1 presents the corresponding values of $c_{q, m}$ for comparison.

\begin{tabular}{|c|ccccc|}
\hline & $m=-1$ & $m=0$ & $m=1$ & $m=2$ & $m=3$ \\
\hline$q=0$ & 0.289 & 0.109 & 0.051 & 0.03 & 0.021 \\
$q=1$ & 0.106 & 0.109 & 0.088 & 0.058 & 0.035 \\
\hline
\end{tabular}

Table 1: Numerical values of $c_{q, m}(f)$ by the QP-approximation for different $q$ and $m$. The value $m=-1$ corresponds to the truncated Fourier series.

\section{Polynomial Corrections}

The conditions of Theorems 3,4 and 6 show the importance of the derivativecondition

$$
f^{(k)}(-1)=f^{(k)}(1)=0, k=0,1,2 \ldots
$$

for the accelerated convergence of the QP-approximations. If the exact values of $f^{(k)}(-1)$ and $f^{(k)}(1), k=0,1,2, \ldots$ are known, then the application of the polynomial correction approach will tremendously improve the convergence.

More specifically, consider the following representation of $f$ (see also [95]) similar to the Lanczos representation

$$
f(x)=h(x)+\sum_{k=0}^{q-1} A_{k}^{-}(f) P_{k, q}(x)+\sum_{k=0}^{q-1} A_{k}^{+}(f) Q_{k, q}(x),
$$


where

$$
A_{k}^{-}(f)=f^{(k)}(1)-f^{(k)}(-1), A_{k}^{+}(f)=f^{(k)}(1)+f^{(k)}(-1),
$$

and $P_{k, q}, Q_{k, q}, k=0, \ldots, q-1$ are 2-periodic polynomials satisfying the following conditions

$$
P_{k, q}^{(s)}(1)-P_{k, q}^{(s)}(-1)=\delta_{k, s}, P_{k, q}^{(s)}(1)+P_{k, q}^{(s)}(-1)=0, k, s=0, \ldots, q-1,
$$

and

$$
Q_{k, q}^{(s)}(1)+Q_{k, q}^{(s)}(-1)=\delta_{k, s}, Q_{k, q}^{(s)}(1)-Q_{k, q}^{(s)}(-1)=0, k, s=0, \ldots, q-1 .
$$

Let us show how polynomials $P_{k, q}(x)$ and $Q_{k, q}(x)$ can be constructed (see $[95]$ ). We put

$$
P_{q-1, q}(x)=\frac{x^{2}\left(x^{2}-1\right)^{q-1}}{2^{q}(q-1) !}, Q_{q-1, q}(x)=\frac{x\left(x^{2}-1\right)^{q-1}}{2^{q}(q-1) !}
$$

if $q$ is even, and

$$
P_{q-1, q}(x)=\frac{x\left(x^{2}-1\right)^{q-1}}{2^{q}(q-1) !}, Q_{q-1, q}(x)=\frac{x^{2}\left(x^{2}-1\right)^{q-1}}{2^{q}(q-1) !}
$$

if $q$ is odd. Then, the polynomials can be represented by the following recurrent relations

$$
\begin{aligned}
& P_{k, q}(x)=P_{k, q-1}(x)-\left(P_{k, q-1}^{(q-1)}(1)+P_{k, q-1}^{(q-1)}(-1)\right) Q_{q-1, q}(x), \\
& Q_{k, q}(x)=Q_{k, q-1}(x)-\left(Q_{k, q-1}^{(q-1)}(1)-Q_{k, q-1}^{(q-1)}(-1)\right) P_{q-1, q}(x)
\end{aligned}
$$

if $q-k$ is even, and

$$
\begin{gathered}
P_{k, q}(x)=P_{k, q-1}(x)-\left(P_{k, q-1}^{(q-1)}(1)-P_{k, q-1}^{(q-1)}(-1)\right) P_{q-1, q}(x), \\
Q_{k, q}(x)=Q_{k, q-1}(x)-\left(Q_{k, q-1}^{(q-1)}(1)+Q_{k, q-1}^{(q-1)}(-1)\right) Q_{q-1, q}(x)
\end{gathered}
$$

if $q-k$ is odd.

Conditions (43) and (44) assure that the function $h$ in 42 satisfies the required derivative-conditions

$$
h^{(k)}(1)=h^{(k)}(-1)=0, k=0, \ldots, q-1 .
$$

Then, the approximation of $h$ by the QP-approximation leads to the following quasi-periodic-polynomial (QPP-) approximation

$$
S_{N, m, q}(f, x)=S_{N, m}(h, x)+\sum_{k=0}^{q-1} A_{k}^{-}(f) P_{k, q}(x)+\sum_{k=0}^{q-1} A_{k}^{+}(f) Q_{k, q}(x),
$$


with the error

$$
R_{N, m, q}(f, x)=f(x)-S_{N, m, q}(f, x) .
$$

The Fourier quasi-periodic coefficients of $h$ can be expressed by means of the corresponding coefficients of the functions $f, P_{k, q}$ and $Q_{k, q}$.

Theorems 3, 4 and 6 can be reformulated for the QPP-approximations by omitting the corresponding derivative-conditions. Let us consider only the analog of Theorem 3 for the QPP-approximations.

Theorem 7 Let $f^{(q+2 m+2)} \in A C[-1,1]$ for some $q, m \geq 0$. Then, the following estimate holds for $|x|<1$

$$
R_{N, m, q}(f, x)=(-1)^{N+m+1} \frac{D_{N, m}(h, x)}{N^{q+m+2}}+o\left(N^{-q-m-2}\right), N \rightarrow \infty,
$$

where $D_{N, m}(f, x)$ is defined in Theorem 3 .

\section{Implementation Notes}

Practical realization of the QPP-approximations is feasible only by efficient approximation of derivatives and calculation of Fourier quasi-periodic coefficients. In general, derivatives at the endpoints are unknown, especially assuming that parameter $q$ in the polynomial correction can tend to infinity. Approximation of the Fourier quasi-periodic coefficients can be performed either by some quadratures (see [124, 125]) if the values of $f$ are known on a grid or via known classical Fourier coefficients on $[-1,1]$ (see [96]).

Let us discuss an approach that leads to QPP-approximations with approximate derivatives with better convergence compared to the ones with the exact derivatives. Similar phenomenon was observed and reported in 86, 87 for the Eckhoff method named as auto-correction phenomenon. Let us return to (9), where unknown coefficients $c_{j}^{l e f t}(f)$ and $c_{j}^{\text {right }}(f), j=0, \ldots, m$ should be found from the system (10). In particular, the latest is one of the main reasons of the fast convergence of the QP-approximation compared to the truncated Fourier series. We will exploit it for approximation of the derivatives. Let us rewrite (10) for the function $h$ in 42 as follows

$$
H_{n, m}=\sum_{j=0}^{m} c_{j}^{l e f t}(h) e^{i \pi n \sigma x_{j}^{*}}+h_{n, m}+\sum_{j=0}^{m} c_{j}^{r i g h t}(h) e^{-i \pi n \sigma x_{j}^{*}} .
$$

Taking into account that

$$
h(x)=f(x)-\sum_{k=0}^{q-1} A_{k}^{-}(f) P_{k, q}(x)-\sum_{k=0}^{q-1} A_{k}^{+}(f) Q_{k, q}(x),
$$


we get

$$
\begin{aligned}
& H_{n, m}=\sum_{j=0}^{m} c_{j}^{l e f t}(f) e^{i \pi n \sigma x_{j}^{*}}+f_{n, m}+\sum_{j=0}^{m} c_{j}^{r i g h t}(f) e^{-i \pi n \sigma x_{j}^{*}} \\
& -\sum_{k=0}^{q-1} A_{k}^{-}(f)\left[\sum_{j=0}^{m} c_{j}^{l e f t}\left(P_{k, q}\right) e^{i \pi n \sigma x_{j}^{*}}+\left(P_{k, q}\right)_{n, m}+\sum_{j=0}^{m} c_{j}^{r i g h t}\left(P_{k, q}\right) e^{-i \pi n \sigma x_{j}^{*}}\right] \\
& -\sum_{k=0}^{q-1} A_{k}^{+}(f)\left[\sum_{j=0}^{m} c_{j}^{l e f t}\left(Q_{k, q}\right) e^{i \pi n \sigma x_{j}^{*}}+\left(Q_{k, q}\right)_{n, m}+\sum_{j=0}^{m} c_{j}^{r i g h t}\left(Q_{k, q}\right) e^{-i \pi n \sigma x_{j}^{*}}\right],
\end{aligned}
$$

where

$$
\begin{gathered}
\left(P_{k, q}\right)_{n, m}=\frac{\sigma}{2} \int_{-1}^{1} P_{k, q}(t) e^{-i \pi n \sigma t} d t,\left(Q_{k, q}\right)_{n, m}=\frac{\sigma}{2} \int_{-1}^{1} Q_{k, q}(t) e^{-i \pi n \sigma t} d t, \\
c_{j}^{\text {left }}\left(P_{k, q}\right)=\frac{\sigma}{4 N} P_{k, q}\left(-x_{j}^{*}+2\right), c_{j}^{\text {right }}\left(P_{k, q}\right)=\frac{\sigma}{4 N} P_{k, q}\left(x_{j}^{*}-2\right), \\
c_{j}^{\text {left }}\left(Q_{k, q}\right)=\frac{\sigma}{4 N} Q_{k, q}\left(-x_{j}^{*}+2\right), c_{j}^{\text {right }}\left(\nu_{k, q}\right)=\frac{\sigma}{4 N} Q_{k, q}\left(x_{j}^{*}-2\right),
\end{gathered}
$$

and, we used 2-periodicity of polynomials $P_{k, q}$ and $Q_{k, q}$. Now, following the idea behind (10), we consider the following system

$$
H_{n, m}=0,|n|=N-m-q, \ldots, N
$$

for determination of unknowns $A_{k}^{-}(f), A_{k}^{+}(f), k=0, \ldots, q-1$ and $c_{j}^{\text {left }}(f)$, $c_{j}^{\text {right }}(f), j=0, \ldots, m$.

One of our future works will be the investigation of the system (47) for its effective solutions. Now, let us numerically investigate its impact on the QPP-approximations for a specific simple function. Let

$$
f(x)=\sin (x-1)
$$

which does not accomplish the required derivative-conditions. Figure 1 shows the absolute values of the errors of the QPP-approximations with the exact values of the derivatives. The left figures correspond to the interval $[-0.8,0.8]$ and the right ones correspond to the entire interval $[-1,1]$. Naturally, the accuracy increases with the values of parameter $q$. Figure 2 corresponds to the QPP-approximations with approximate derivatives from the system (47). The comparison of the figures reveals the essence of the auto-correction phenomenon. Inside the interval of approximation, QPPapproximations with approximate jumps have better accuracies compared to the QPP-approximations with the exact jumps. For $m=2$ and $q=1$, 
the maximum of the absolute error is $3,5 \cdot 10^{-7}$ versus $1,4 \cdot 10^{-6}$. For $m=2$ and $q=4$, the maximum of the absolute error is $2 \cdot 10^{-12}$ versus $1,5 \cdot 10^{-9}$. As larger is the value of $q$ as bigger is the difference between approximations via exact and numeric derivatives. It is important, that the QPP-approximations with approximate derivatives have improved accuracy on the entire interval as well. For example, we see $6 \cdot 10^{-7}$ versus $1,2 \cdot 10^{-6}$ for $m=2$ and $q=4$. We did not observe it in the case of the Eckhoff method. There, the improvements were observed only away from the singularities $x= \pm 1$. However, it is easy to explain for the QPP-approximations as interval $[-1,1]$ is an inside interval for the extended $[-1 / \sigma, 1 / \sigma]$ interval where the approximations are applied. One of our future works should be theoretical investigation of the auto-correction phenomenon for the QPPapproximations.

\section{Conclusion}

The paper continued investigations of the quasi-periodic (QP-) approximations initiated in 96 . The main goal was a detailed investigation of Algorithm $\mathrm{C}$ proposed in [96], which led to the explicit realization of the approximations through the inverse of a Vandermonde matrix. We explored the convergence of the approximations in different frameworks and derived exact constants for the corresponding asymptotic errors.

The main results were revealed in Theorems 3 , 4 and 6. The estimates of those theorems showed that the convergence rates of the approximations depended on the following derivative-conditions at the endpoints of $[-1,1]$

$$
f^{(k)}(1)=f^{(k)}(-1)=0, k=0, \ldots, q-1 .
$$

Those requirements were stricter compared to the classical requirements for the Fourier expansions (actually meaning periodic and smooth continuation of the function $f$ from $[-1,1]$ onto the real line $\mathbb{R}$ )

$$
f^{(k)}(1)=f^{(k)}(-1), k=0, \ldots, q-1 .
$$

Theorem 3 revealed the pointwise behavior of the approximations away from the endpoints. It showed convergence rate $O\left(N^{-q-m-2}\right)$ as $N \rightarrow \infty$, while for the truncated Fourier series (see [86], Theorem 2.4), the convergence rate was $O\left(N^{-q-1}\right)$ which formally corresponded to the estimate of Theorem 3 with $m=-1$. We observed improvement in the convergence rate by factor $O\left(N^{m+1}\right)$, although with different smoothness requirements and derivative-conditions.

Theorem 4 estimated the convergence rate of the QP-approximations at $x= \pm 1$. It showed $O\left(N^{-q}\right)$ as $N \rightarrow \infty$. Hence, the QP-approximations diverged for $q=0$ at $x= \pm 1$. 

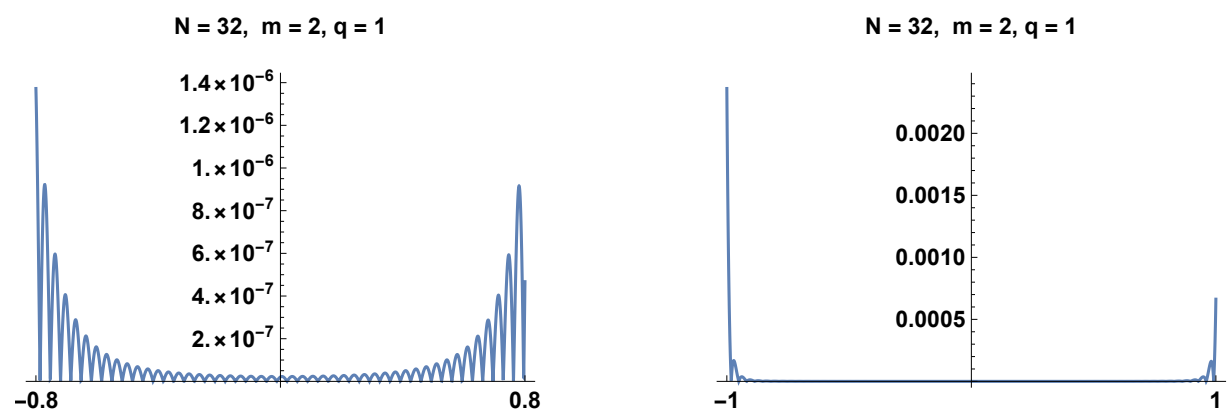

$\mathrm{N}=32, \mathrm{~m}=2, \mathrm{q}=\mathbf{2}$
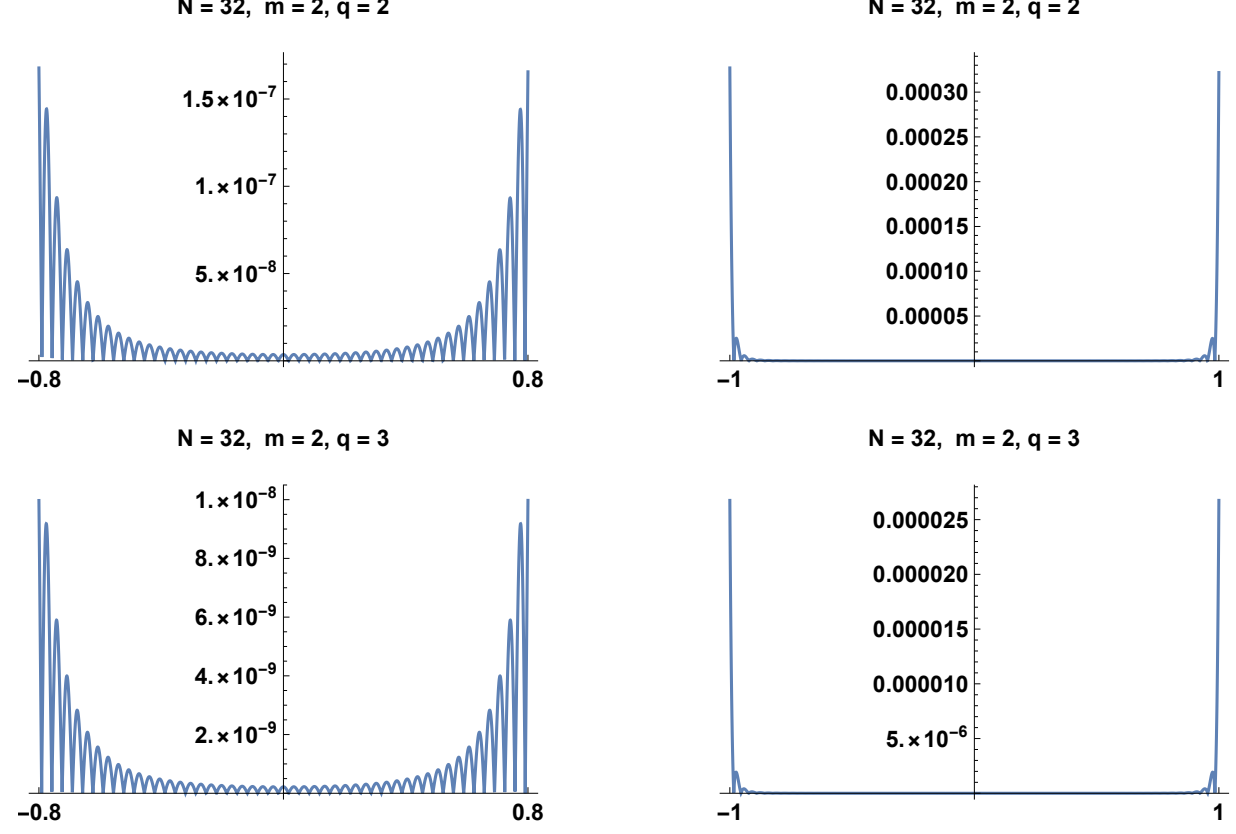

$\mathrm{N}=32, \mathrm{~m}=2, \mathrm{q}=4$

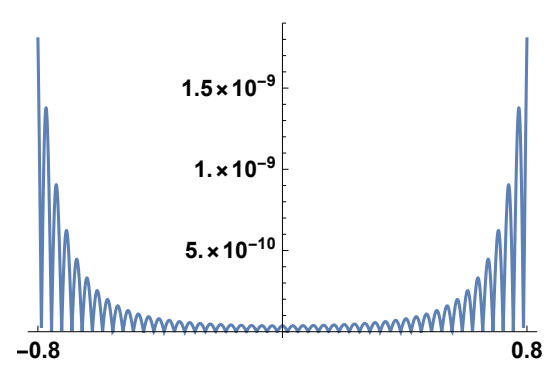

$\mathrm{N}=32, \mathrm{~m}=2, \mathrm{q}=4$

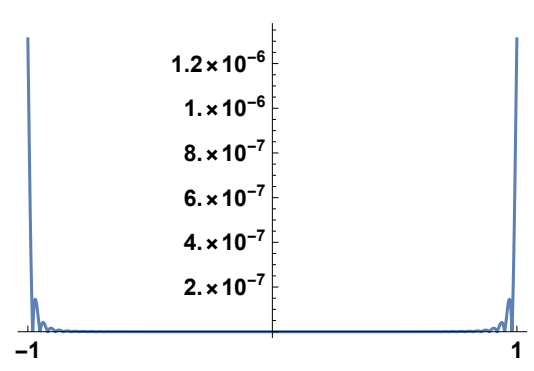

Figure 1: QPP-approximations with the exact values of the endpoint derivatives.

Theorem 6 explored the convergence in the framework of $L_{2}[-1,1]$-norm. It showed identical convergence rate $O\left(N^{-q-\frac{1}{2}}\right)$ as for the truncated Fourier series. The benefit of the QP-approximation compared to the truncated Fourier series was in smaller constant $c_{q, m}(f), m \geq 0$ compared to $c_{q,-1}(f)$ which was based on experimental evidence without the theoretical proof. 

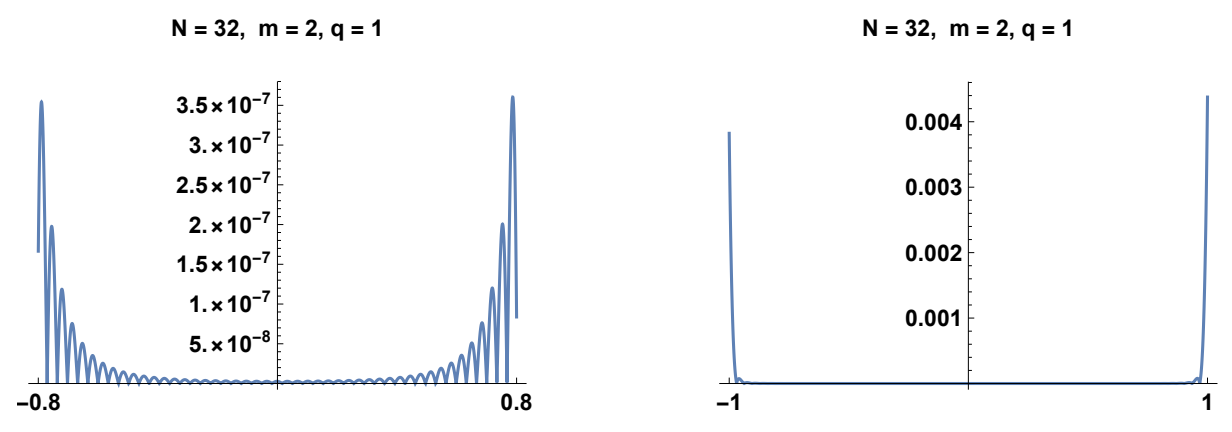

$\mathrm{N}=32, \mathrm{~m}=2, \mathrm{q}=\mathbf{2}$

$\mathrm{N}=32, \mathrm{~m}=2, \mathrm{q}=2$
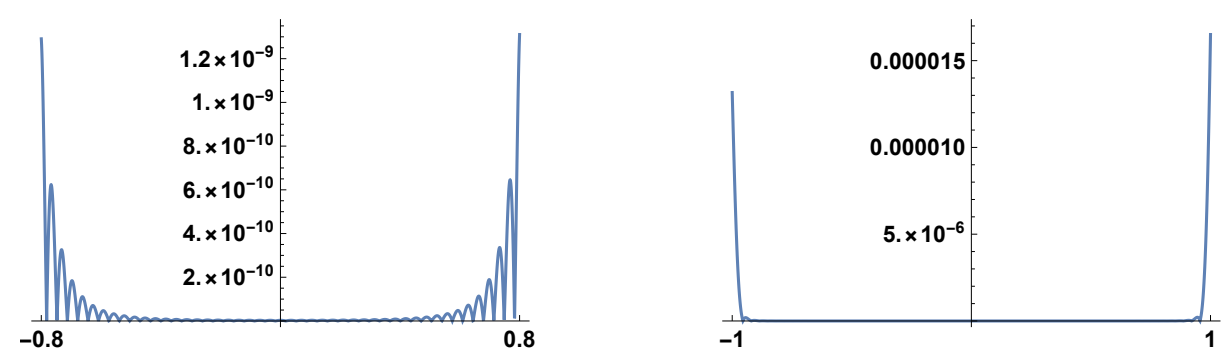

$\mathrm{N}=32, \mathrm{~m}=2, \mathrm{q}=3$

$\mathrm{N}=32, \mathrm{~m}=2, \mathrm{q}=3$
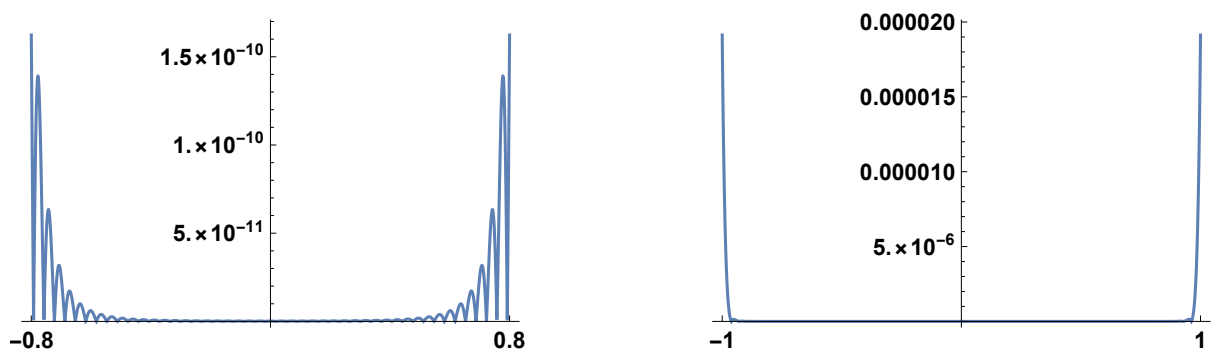

$\mathrm{N}=32, \mathrm{~m}=2, \mathrm{q}=4$

$\mathrm{N}=32, \mathrm{~m}=2, \mathrm{q}=4$
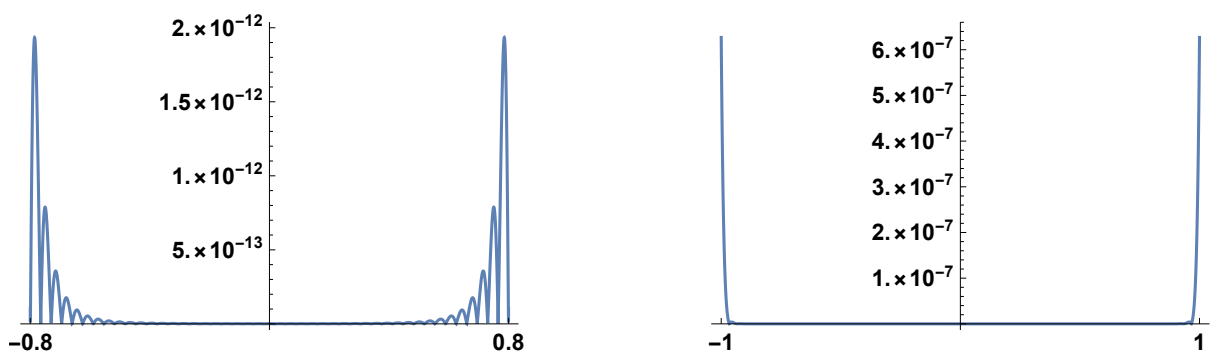

Figure 2: QPP-approximations with the approximated values of the endpoint derivatives.

Additional convergence acceleration was achieved via application of the well-known polynomial correction method (QPP-approximations). We showed the system of linear equations (see (47)) for approximation of the coefficients $c_{j}^{\text {left }}(f)$ and $c_{j}^{\text {right }}(f)$ in 46 as well as for determination of derivatives $A_{k}^{-}(f)$ 
and $A_{k}^{+}(f)$ in 42 . The approach led to the improved convergence of the QPP-approximations with approximated derivatives compared to the ones that exploited the exact derivatives. Similar improvement was detected for the Eckhoff method and was named as auto-correction phenomenon. In case of the QPP-approximations, the improvement was noticed both on the entire interval and much severe away from the endpoint-singularities.

\section{Acknowledgement}

We thank anonymous reviewers for careful and critical reading of our paper. Their thoughtful comments tremendously helped to improve our manuscript.

\section{References}

[1] J. W. Gibbs, Fourier's series, Letter in Nature, 59 (1899), pp. 200-200.

[2] M. Bôcher, Introduction to the theory of Fourier's series, Ann. of Math. (2), 7 (1906), no. 3, pp. 81-152.

[3] E. Hewitt and R. E. Hewitt, The Gibbs-Wilbraham phenomenon: an episode in Fourier analysis, Arch. Hist. Exact Sci., 21 (1979/80), no. 2, pp. 129-160. https://doi.org/10.1007/bf00330404

[4] A. J. Jerri, The Gibbs phenomenon in Fourier analysis, splines and wavelet approximations, vol. 446 of Mathematics and its Applications, Dordrecht: Kluwer Academic Publishers, 1998. https://doi.org/10.1007/978-1-4757-2847-7_4

[5] A. Zygmund, Trigonometric Series. Vol. 1,2, Cambridge Univ. Press, 1959.

[6] L. Fejer, Untersuchungen über Fouriersche reihen, Math. Ann., 58 (1904), pp. 51-69.

[7] K. Knopp, Infinite sequences and series, New York: Dover Publications Inc., 1956.

[8] P. Wynn, On a device for computing the $e_{m}\left(S_{n}\right)$ tranformation, Math. Tables Aids Comput., 10 (1956), pp. 91-96.

[9] A. Haug, Theoretical solid state physics, New York: Pergamon Press Oxford, 1972. 
[10] D. Levin, Development of non-linear transformations of improving convergence of sequences, Internat. J. Comput. Math., 3 (1973), pp. 371388.

[11] A. Majda, J. McDonough, and S. Osher, The Fourier method for nonsmooth initial data, Math. Comp., 32 (1978), no. 144, pp. 1041-1081. https://doi.org/10.1090/s0025-5718-1978-0501995-4

[12] J. Wimp, Sequence transformations and their applications, vol. 154 of Mathematics in Science and Engineering, New York: Academic Press, 1981.

[13] D. A. Smith and W. F. Ford, Numerical comparisons of nonlinear convergence accelerators, Math. Comp., 38 (1982), no. 158, pp. 481499. https://doi.org/10.1090/s0025-5718-1982-0645665-1

[14] S. Biringen and K. H. Kao, On the application of pseudospectral FFT techniques to nonperiodic problems, Internat. J. Numer. Methods Fluids, 9 (1989), no. 10, pp. 1235-1267. https://doi.org/10.1002/fld.1650091006

[15] J. P. Boyd, Sum-accelerated pseudospectral methods: the Euleraccelerated sinc algorithm, Appl. Numer. Math., 7 (1991), no. 4, pp. 287-296. https://doi.org/10.1016/0168-9274(91)90065-8

[16] H. Vandeven, Family of spectral filters for discontinuous problems, J. Sci. Comput., 6 (1991), no. 2, pp. 159-192. https://doi.org/10.1007/bf01062118

[17] C. Brezinski and M. Redivo Z., Extrapolation methods, vol. 2 of Studies in Computational Mathematics, Amsterdam: North-Holland Publishing Co., 1991.

[18] D. Gottlieb, C.-W. Shu, A. Solomonoff, and H. Vandeven, On the Gibbs phenomenon. I. Recovering exponential accuracy from the Fourier partial sum of a nonperiodic analytic function, J. Comput. Appl. Math., 43 (1992), no. 1-2, pp. 81-98. https://doi.org/10.1016/0377-0427(92)90260-5

[19] D. Gottlieb, Issues in the application of high order schemes, in Algorithmic trends in computational fluid dynamics (1991), ICASE/NASA LaRC Ser., pp. 195-218, New York: Springer, 1993. https://doi.org/10.1007/978-1-4612-2708-3_12

[20] H. H. H. Homeier, Some applications of nonlinear convergence accelerators, IJQC, 45 (1993), no. 6, pp. 545-562. 
[21] J. P. Boyd, A lag-averaged generalization of Euler's method for accelerating series, Appl. Math. Comput., 72 (1995), no. 2-3, pp. 143-166. https://doi.org/10.1016/0096-3003(94)00180-c

[22] A. Dillmann and G. Grabitz, On a method to evaluate Fourier-Bessel series with poor convergence properties and its application to linearized supersonic free jet flow, Quart. Appl. Math., 53 (1995), no. 2, pp. 335-352. https://doi.org/10.1090/qam/1330656

[23] D. Gottlieb and C.-W. Shu, On the Gibbs phenomenon. IV. Recovering exponential accuracy in a subinterval from a Gegenbauer partial sum of a piecewise analytic function, Math. Comp., 64 (1995), no. 211, pp. 1081-1095. https://doi.org/10.1090/s0025-5718-1995-1284667-0

[24] D. Gottlieb and C.-W. Shu, On the Gibbs phenomenon. V. Recovering exponential accuracy from collocation point values of a piecewise analytic function, Numer. Math., 71 (1995), no. 4, pp. 511-526. https://doi.org/10.1007/s002110050155

[25] D. Gottlieb and C.-W. Shu, On the Gibbs phenomenon. III. Recovering exponential accuracy in a sub-interval from a spectral partial sum of a piecewise analytic function, SIAM J. Numer. Anal., 33 (1996), no. 1, pp. 280-290. https://doi.org/10.1137/0733015

[26] L. Vozovoi, M. Israeli, and A. Averbuch, Analysis and application of Fourier-Gegenbauer method to stiff differential equations, SIAM J. Numer. Anal., 33 (1996), no. 5, pp. 1844-1863. https://doi.org/10.1137/s0036142994263591

[27] C. Oleksy, A convergence acceleration method of Fourier series, Comput. Phys. Comm., 96 (1996), no. 1, pp. 17-26.

[28] J. Geer and N. S. Banerjee, Exponentially accurate approximations to piece-wise smooth periodic functions, J. Sci. Comput., 12 (1997), no. 3, pp. 253-287.

[29] A. Gelb and D. Gottlieb, The resolution of the Gibbs phenomenon for 'spliced' functions in one and two dimensions, Computers Math. Applic., 33 (1997), no. 11, pp. 35-58.

[30] D. Gottlieb and C.-W. Shu, On the Gibbs phenomenon and its resolution, SIAM Rev., 39 (1997), no. 4, pp. 644-668. https://doi.org/10.1137/s0036144596301390

[31] L. Vozovoi, A. Weill, and M. Israeli, Spectrally accurate solution of nonperiodic differential equations by the Fourier-Gegenbauer 
method, SIAM J. Numer. Anal., 34 (1997), no. 4, pp. 1451-1471. https://doi.org/10.1137/s0036142994278814

[32] J. P. Boyd, Two comments on filtering (artificial viscosity) for Chebyshev and Legendre spectral and spectral element methods: preserving boundary conditions and interpretation of the filter as a diffusion, J. Comput. Phys., 143 (1998), no. 1, pp. 283-288. https://doi.org/10.1006/jcph.1998.5961

[33] G. Kvernadze, Determination of the jumps of a bounded function by its Fourier series, J. Approx. Theory, 92 (1998), no. 2, pp. 167-190. https://doi.org/10.1006/jath.1997.3125

[34] S. L. Marshall, Convergence acceleration of Fourier series by analytical and numerical application of Poisson's formula, J. Phys. A, 31 (1998), no. 11, pp. 2691-2704. https://doi.org/10.1088/0305-4470/31/11/016

[35] A. Gelb and E. Tadmor, Detection of edges in spectral data, Appl. Comput. Harmon. Anal., 7 (1999), no. 1, pp. 101-135.

[36] A. Gelb and E. Tadmor, Detection of edges in spectral data. II. Nonlinear enhancement, SIAM J. Numer. Anal., 38 (2000), no. 4, pp. 13891408. https://doi.org/10.1137/s0036142999359153

[37] G. Kvernadze, T. Hagstrom, and H. Shapiro, Detecting the singularities of a function of $V_{p}$ class by its integrated Fourier series, Comput. Math. Appl., 39 (2000), no. 9-10, pp. 25-43. https://doi.org/10.1016/s0898-1221(00)00084-5

[38] A. Gelb, A hybrid approach to spectral reconstruction of piecewise smooth functions, J. Sci. Comput., 15 (2000), no. 3, pp. 293-322.

[39] H. N. Mhaskar and J. Prestin, On the detection of singularities of a periodic function, Adv. Comput. Math., 12 (2000), no. 2-3, pp. 95131.

[40] R. K. Wright, A robust method for accurately representing non-periodic functions given Fourier coefficient information, J. Comput. and Appl. Math., 140 (2002), no. 1, pp. 837-848. https://doi.org/10.1016/s0377-0427(01)00518-0

[41] E. Tadmor and J. Tanner, Adaptive mollifiers for high resolution recovery of piecewise smooth data from its spectral information, Found. Comput. Math., 2 (2002), no. 2, pp. 155-189. https://doi.org/10.1007/s102080010019 
[42] A. Gelb and E. Tadmor, Spectral reconstruction of piecewise smooth functions from their discrete data, Math. Model. Numer. Anal., 36 (2002), no. 2, pp. 155-175. https://doi.org/10.1051/m2an:2002008

[43] J.-H. Jung and B. D. Shizgal, Generalization of the inverse polynomial reconstruction method in the resolution of the Gibbs phenomenon, J. Comput. Appl. Math., 172 (2004), no. 1, pp. 131-151. https://doi.org/10.1016/j.cam.2004.02.003

[44] R. K. Wright, Local spline approximation of discontinuous functions and location of discontinuities, given low-order Fourier coefficient information, J. Comput. and Appl. Math., 164-165 (2004), pp. 783-795. https://doi.org/10.1016/s0377-0427(03)00647-2

[45] R. Archibald, A. Gelb, and J. Yoon, Polynomial fitting for edge detection in irregularly sampled signals and images, SIAM J. Numer. Anal., 43 (2005), no. 1, pp. 259-279. https://doi.org/10.1137/s0036142903435259

[46] J.-H. Jung and B. D. Shizgal, Inverse polynomial reconstruction of two dimensional Fourier images, J. Sci. Comput., 25 (2005), no. 3, pp. 367-399. https://doi.org/10.1007/s10915-004-4795-3

[47] E. Tadmor and J. Tanner, Adaptive filters for piecewise smooth spectral data, IMA J. Numer. Anal., 25 (2005), no. 4, pp. 635-647. https://doi.org/10.1093/imanum/dri026

[48] A. Nersessian and A. Poghosyan, Accelerating the convergence of trigonometric series, Cent. Eur. J. Math., 4 (2006), no. 3, pp. 435448. https://doi.org/10.2478/s11533-006-0016-7

[49] J.-H. Jung and B. D. Shizgal, On the numerical convergence with the inverse polynomial reconstruction method for the resolution of the Gibbs phenomenon, J. Comput. Phys., 224 (2007), no. 2, pp. 477-488. https://doi.org/10.1016/j.jcp.2007.01.018

[50] S. Paszkowski, Convergence acceleration of orthogonal series, Numer. Algorithms, 47 (2008), no. 1, pp. 35-62. https://doi.org/10.1007/s11075-007-9146-7

[51] J. P. Boyd, Large-degree asymptotics and exponential asymptotics for Fourier, Chebyshev and Hermite coefficients and Fourier transforms, J. Engrg. Math., 63 (2009), no. 2-4, pp. 355-399. https://doi.org/10.1007/s10665-008-9241-3 
[52] B. Adcock, Gibbs phenomenon and its removal for a class of orthogonal expansions, BIT, 51 (2011), no. 1, pp. 7-41. https://doi.org/10.1007/s10543-010-0301-5

[53] A. Poghosyan, On a convergence of the Fourier-Pade approximation, Armen. J. Math., 4 (2012), no. 2, pp. 49-79.

[54] A. Poghosyan, On the convergence of rational-trigonometricpolynomial approximations realized by roots of Laguerre polynomials, Izv. Nats. Akad. Nauk Armenii Mat., 48 (2013), no. 6, pp. 82-91. https://doi.org/10.3103/s1068362313060101

[55] A. Poghosyan, On a convergence of the Fourier-Pade interpolation, Armen. J. Math., 5 (2013), no. 1, pp. 1-25.

[56] A. Nersessian, On some fast implementations of Fourier interpolation, in Operator Theory and Harmonic Analysis (A. N. Karapetyants, V. V. Kravchenko, E. Liflyand, and H. R. Malonek, eds.), pp. 463-477, Springer International Publishing, 2021. https://doi.org/10.1007/9783-030-77493-6_27

[57] A. Krylov, Lectures on approximate calculations (lectures delivered in 1906 in Russian). St. Petersburg: Tipolitography of Birkenfeld, 1907.

[58] G. P. Tolstov, Fourier series. Translated from the Russian by Richard A. Silverman, Englewood Cliffs, N.J.: Prentice-Hall Inc., 1962.

[59] C. Lanczos, Discourse on Fourier series. Edinburgh: Oliver and Boyd, 1966.

[60] W. B. Jones and G. Hardy, Accelerating convergence of trigonometric approximations, Math. Comp., 24 (1970), pp. 547-560. https://doi.org/10.1090/s0025-5718-1970-0277086-x

[61] J. N. Lyness, Computational techniques based on the Lanczos representation, Math. Comp., 28 (1974), pp. 81-123. https://doi.org/10.1090/s0025-5718-1974-0334458-6

[62] A. Poghosyan, Asymptotic behavior of the Krylov-Lanczos interpolation, Anal. Appl. (Singap.), 7 (2009), no. 2, pp. 199-211. https://doi.org/10.1142/s0219530509001359

[63] A. Poghosyan, On a pointwise convergence of trigonometric interpolations with shifted nodes, Armen. J. Math., 5 (2013), no. 2, pp. 105-122.

[64] D. Gottlieb and S. A. Orszag, Numerical analysis of spectral methods: theory and applications. Philadelphia, Pa.: Society for Industrial and Applied Mathematics, 1977. 
[65] G. Baszenski and F.-J. Delvos, Error estimates for sine series expansions, Math. Nachr., 139 (1988), pp. 155-166. https://doi.org/10.1002/mana.19881390114

[66] P. J. Roache, A pseudo-spectral FFT technique for non-periodic problems, J. Comput. Phys., 27 (1978), no. 2, pp. 204-220. https://doi.org/10.1016/0021-9991(78)90005-0

[67] D. Gottlieb, L. Lustman, and S. A. Orszag, Spectral calculations of one-dimensional inviscid compressible flows, SIAM J. Sci. Statist. Comput., 2 (1981), no. 3, pp. 296-310. https://doi.org/10.1137/0902024

[68] S. Abarbanel and D. Gottlieb, Information content in spectral calculations, in Progress and supercomputing in computational fluid $d y$ namics (Jerusalem, 1984), 6 of Progr. Sci. Comput., pp. 345-356, Boston, MA: Birkhäuser Boston, 1985. https://doi.org/10.1007/9781-4612-5162-0_18

[69] S. Abarbanel, D. Gottlieb, and E. Tadmor, Spectral methods for discontinuous problems, in Numerical methods for fluid dynamics, II (Reading, 1985), 7 of Inst. Math. Appl. Conf. Ser. New Ser., pp. 129153, New York: Oxford Univ. Press, 1986.

[70] W. Cai, D. Gottlieb, and C. W. Shu, Essentially non oscillatory spectral Fourier methods for shock wave calculations, Math. Comp., 52 (1989), pp. 389-410. https://doi.org/10.1090/s0025-5718-19890955749-2

[71] D. Gottlieb, Spectral methods for compressible flow problems, in Ninth international conference on numerical methods in fluid dynamics (Saclay, 1984), 218 of Lecture Notes in Phys., pp. 48-61, Berlin: Springer, 1985. https://doi.org/10.1007/3-540-13917-6_109

[72] S. A. Orszag, Numerical simulation of incompressible flows within simple boundaries. I. Galerkin (spectral) representations, Studies in Appl. Math., 50 (1971), pp. 293-327. https://doi.org/10.1002/sapm1971504293

[73] H. Wengle and J. H. Seinfeld, Pseudospectral solution of atmospheric diffusion problems, J. Computational Phys., 26 (1978), no. 1, pp. 87106. https://doi.org/10.1016/0021-9991(78)90101-8

[74] K. S. Eckhoff, Accurate and efficient reconstruction of discontinuous functions from truncated series expansions, Math. Comp., 61 (1993), pp. 745-763. https://doi.org/10.1090/s0025-5718-1993-1195430-1 
[75] K. S. Eckhoff, Accurate reconstructions of functions of finite regularity from truncated Fourier series expansions, Math. Comp., 64, pp. 671690. https://doi.org/10.1090/s0025-5718-1995-1265014-7

[76] K. S. Eckhoff, On a high order numerical method for functions with singularities, Math. Comp., 67 (1998), pp. 1063-1087. https://doi.org/10.1090/s0025-5718-98-00949-1

[77] A. Sidi, Practical extrapolation methods, 10 of Cambridge Monographs on Applied and Computational Mathematics. Cambridge: Cambridge University Press, 2003.

[78] J. K. Shaw, L. W. Johnson, and R. D. Riess, Accelerating convergence of eigenfunction expansions, Math. Comp., 30 (1976), no. 135, pp. 469-477. https://doi.org/10.1090/s0025-5718-1976-0418404-4

[79] G. Baszenski, F.-J. Delvos, and M. Tasche, A united approach to accelerating trigonometric expansions, Computers Math. Applic., 30 (1995), no. 3-6, pp. 33-49. https://doi.org/10.1016/08981221(95)00084-4

[80] M. Tasche, Zur Konvergenzbeschleunigung von FourierReihen, Math. Nachr., $90 \quad$ (1979), pp. 123-134. https://doi.org/10.1002/mana.19790900110

[81] A. Barkhudaryan, R. Barkhudaryan, and A. Poghosyan, Asymptotic behavior of Eckhoff's method for Fourier series convergence acceleration, Anal. Theory Appl., 23 (2007), no. 3, pp. 228-242. https://doi.org/10.1007/s10496-007-0228-0

[82] A. Nersessian and A. Poghosyan, Convergence acceleration of twodimensional Fourier interpolation, Armen. J. Math., 1 (2008), no. 1, pp. 50-63.

[83] J. P. Boyd, Acceleration of algebraically-converging Fourier series when the coefficients have series in powers in $1 / n$, J. Comput. Phys., 228 (2009), no. 5, pp. 1404-1411, 2009. https://doi.org/10.1016/j.jcp.2008.10.039

[84] A. Poghosyan, Asymptotic behavior of the Eckhoff method for convergence acceleration of trigonometric interpolation, Anal. Theory Appl., 26 (2010), no. 3, pp. 236-260. https://doi.org/10.1007/s10496-0100236-3

[85] A. Nersessian, Convergence acceleration of Fourier series revisited, Armen. J. Math., 3 (2010), no. 4, pp. 152-161. 
[86] A. Poghosyan, On an auto-correction phenomenon of the KrylovGottlieb-Eckhoff method, IMA J. Numer. Anal., 31 (2011), no. 2, pp. 512-527. https://doi.org/10.1093/imanum/drp043

[87] A. Poghosyan, On an auto-correction phenomenon of the Eckhoff interpolation, Aust. J. Math. Anal. Appl., 9 (2012), no. 1, pp. 1-31.

[88] A. Poghosyan, Asymptotic behavior of the Eckhoff approximation in bivariate case, Anal. Theory Appl., 28 (2012), no. 4, pp. 329-362.

[89] D. Batenkov, N. Sarig, and Y. Yomdin, An 'algebraic' reconstruction of piecewise-smooth functions from integral measurements, Functional Differential Equations, 19 (2012), no. 1-2, pp. 13-30.

[90] A. Nersessian and N. Oganesyan, Quasiperiodic interpolation, Reports of NAS RA, 101 (2001), no. 2, pp. 115-121.

[91] L. Poghosyan and A. Poghosyan, On the pointwise convergence of a quasiperiodic trigonometric interpolation, Izv. Nats. Akad. Nauk Armenii Mat., 49 (2014), no. 3, pp. 68-80.

[92] L. Poghosyan and A. Poghosyan, Asymptotic estimates for the quasiperiodic interpolations, Armen. J. Math., 5 (2013), no. 1, pp. 34-57.

[93] L. Poghosyan, On $L_{2}$-convergence of the quasi-periodic interpolation, Dokl. Nats. Akad. Nauk Armen., 113 (2013), no. 3, pp. 240-247.

[94] A. Poghosyan and L. Poghosyan, On a pointwise convergence of quasi-periodic-rational trigonometric interpolation, Int. J. Anal., 2014 (2014), no. Art. ID 249513, pp. 1-10. https://doi.org/10.1155/2014/249513

[95] L. Poghosyan, Convergence acceleration of quasi-periodic and quasiperiodic-rational interpolations by polynomial corrections, Armen. J. Math., 5 (2013), no. 2, pp. 123-138.

[96] A. Poghosyan, L. Poghosyan, and R. Barkhudaryan, On some quasiperiodic approximations, Armen. J. Math., 12 (2020), no. 10, pp. 1-27.

[97] A. Nersessian, On an over-convergence phenomenon for Fourier series. Basic approach, Armen. J. Math., 10 (2018), no. 9, pp. 1-22.

[98] A. Nersessian, A correction to the article 'On an over-convergence phenomenon for Fourier series. Basic approach', Armen. J. Math., 11 (2019), no. 1, pp. 1-2.

[99] A. Nersessian, A fast method for numerical realization of Fourier tools, IntechOpen, 2020. 
[100] A. Iserles and S. P. Nørsett, From high oscillation to rapid approximation. I. Modified Fourier expansions, IMA J. Numer. Anal., 28 (2008), no. 4, pp. 862-887. https://doi.org/10.1093/imanum/drn006

[101] A. Iserles and S. P. Nørsett, From high oscillation to rapid approximation III: Multivariate expansions, IMA J. Numer. Anal., 29 (2009), no. 4, pp. 882-916. https://doi.org/10.1093/imanum/drn020

[102] M. G. Krein, On a special class of differential operators, Doklady AN USSR, 2 (1935), pp. 345-349.

[103] S. Olver, On the convergence rate of a modified Fourier series, Math. Comp., 78 (2009), no. 267, pp. 1629-1645. https://doi.org/10.1090/s0025-5718-09-02204-2

[104] B. Adcock, Univariate modified Fourier methods for second order boundary value problems, BIT, 49 (2009), no. 2, pp. 249-280. https://doi.org/10.1007/s10543-009-0224-1

[105] B. Adcock, Modified Fourier expansions: theory, construction and applications, PhD Thesis, University of Cambridge, 2010.

[106] B. Adcock, Multivariate modified Fourier series and application to boundary value problems, Numer. Math., 115 (2010), no. 4, pp. 511552. https://doi.org/10.1007/s00211-010-0287-6

[107] B. Adcock, Convergence acceleration of modified Fourier series in one or more dimensions, Math. Comp., 80 (2011), no. 273, pp. 225-261. https://doi.org/10.1090/s0025-5718-2010-02393-2

[108] D. Huybrechs, A. Iserles, and S. P. Nørsett, From high oscillation to rapid approximation IV: Accelerating convergence, IMA J. Numer. Anal., 31 (2011), no. 2, pp. 442-468. https://doi.org/10.1093/imanum/drp046

[109] T. Bakaryan, On a convergence of the modified Fourier-Pade approximations, Armen. J. Math., 8 (2016), no. 2, pp. 120-144.

[110] T. Bakaryan, On a convergence of rational approximations by the modified Fourier basis, Armen. J. Math., 9 (2017), no. 2, pp. 68-83.

[111] A. Poghosyan and T. Bakaryan, Optimal rational approximations by the modified Fourier basis, Abstr. Appl. Anal., 2018 (2018), pp. 1-21. https://doi.org/10.1155/2018/1705409

[112] A. Poghosyan and T. Bakaryan, On interpolation with respect to a modified trigonometric system, Izv. Nats. Akad. Nauk Armenii Mat., 53 (2018), no. 3, pp. 72-83. 
[113] J. P. Boyd, A comparison of numerical algorithms for Fourier extension of the first, second, and third kinds, J. Comput. Phys., 178 (2002), no. 1, pp. 118-160. https://doi.org/10.1006/jcph.2002.7023

[114] O. P. Bruno, Y. Han, and M. M. Pohlman, Accurate, high-order representation of complex three-dimensional surfaces via Fourier continuation analysis, J. Comput. Phys., 227 (2007), no. 2, p. 1094-1125. https://doi.org/10.1016/j.jcp.2007.08.029

[115] D. Huybrechs, On the Fourier extension of nonperiodic functions, SIAM J. Numer. Anal., 47 (2010), no. 6, pp. 4326-4355. https://doi.org/10.1137/090752456

[116] B. Adcock and D. Huybrechs, On the resolution power of Fourier extensions for oscillatory functions, J. Comput. Appl. Math., 260 (2014), pp. 312-336. https://doi.org/10.1016/j.cam.2013.09.069

[117] B. Adcock, D. Huybrechs, and J. Martín-Vaquero, On the numerical stability of Fourier extensions, Found. Comput. Math., 14 (2014), pp. 635-687. https://doi.org/10.1007/s10208-013-9158-8

[118] D. Batenkov and Y. Yomdin, Algebraic Fourier reconstruction of piecewise smooth functions, Math. Comp., 81 (2012), no. 277, pp. 277-318. https://doi.org/10.1090/s0025-5718-2011-02539-1

[119] D. Batenkov, Complete algebraic reconstruction of piecewise-smooth functions from Fourier data, Math. Comp., 84 (2015), no. 295, pp. 2329-2350. https://doi.org/10.1090/s0025-5718-2015-02948-2

[120] A. Björk and V. Pereyra, Solution of Vandermonde systems of equations, Math. Comp., 24 (1970), pp. 893-904.

[121] N. J. Higham, Error analysis of the Björck-Pereyra algorithms for solving Vandermonde systems, Numer. Math., 50 (1987), no. 5, pp. 613632. https://doi.org/10.1007/bf01408579

[122] J. L. López and N. M. Temme, Two-point Taylor expansions of analytic functions, Stud. Appl. Math., 109 (2002), no. 4, pp. 297-311. https://doi.org/10.1111/1467-9590.00225

[123] J. Riordan, Combinatorial identities. New York: John Wiley \& Sons Inc., 1968.

[124] A. Iserles and S. P. Nø rsett, Efficient quadrature of highly oscillatory integrals using derivatives, Proc. R. Soc. Lond. Ser. A Math. Phys. Eng. Sci., 461 (2005), no. 2057, pp. 1383-1399. https://doi.org/10.1098/rspa.2004.1401 
[125] A. Deaño, D. Huybrechs, and A. Iserles, Computing highly oscillatory integrals. Society for Industrial and Applied Mathematics (SIAM), Philadelphia, PA, 2018. https://doi.org/10.1137/1.9781611975123

Arnak V. Poghosyan

Institute of Mathematics,

National Academy of Sciences of Armenia

Bagramian ave. 24/5, 0019 Yerevan, Armenia.

arnak@instmath.sci.am

Lusine D. Poghosyan

Institute of mathematics,

National Academy of Sciences of Armenia

Bagramian ave. 24/5, 0019 Yerevan, Armenia.

lusine@instmath.sci.am

Rafayel H. Barkhudaryan

Institute of mathematics,

National Academy of Sciences of Armenia

Bagramian ave. 24/5, 0019 Yerevan, Armenia.

Yerevan State University,

Alex Manoogian 1, 0025 Yerevan, Armenia.

rafayel@instmath.sci.am

Please, cite to this paper as published in

Armen. J. Math., V. 13, N. 10(2021), pp. 144

https://doi.org/10.52737/18291163-2021.13.10-1-44 